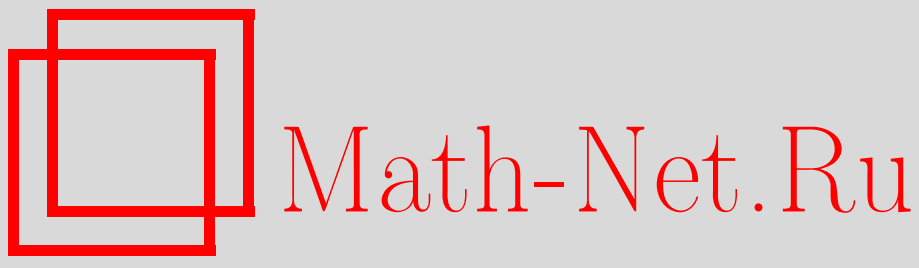

А. В. Болсинов, П. Х. Рихтер, А. Т. Фоменко, Метод круговых молекул и топология волчка Ковалевской, Матем. сб., 2000, том 191, номер 2, 3-42

DOI: https://doi.org/10.4213/sm451

Использование Общероссийского математического портала Math-Net.Ru подразумевает, что вы прочитали и согласны с пользовательским соглашением

http://www . mathnet.ru/rus/agreement

Параметры загрузки:

IP : 54.197 .217 .227

26 апреля 2023 г., $17: 39: 10$ 
УДК $513.944+515.1$

\author{
А.В. Болсинов, П. Рихтер, А.Т. Фоменко
}

\title{
Метод круговых молекул и топология волчка Ковалевской
}

\begin{abstract}
В работе предложен метод вычисления топологических инвариантов слоения фазового пространства на инвариантные торы Лиувилля в случае интегрируемых гамильтоновых систем с двумя степенями свободы. Полностью описана структура этого слоения для интегрируемого случая Ковалевской в динамике твердого тела.
\end{abstract}

Библиография: 32 названия.

\section{§1. Введение}

Волчок Ковалевской - одна из самых удивительных интегрируемых систем классической механики. Его открытие в 1889 году Софьей Васильевной Ковалевской [1] стало важным событием в истории комплексного анализа, лидером которого в то время был ее учитель Карл Вейерштрасс [2].

Эта система не допускает разделения переменных на конфигурационном пространстве, являющемся после редукции по $S^{1}$-симметрии относительно гравитационной оси сферой Пуассона. Тем не менее Ковалевской удалось не только найти “четвертый интеграл” в шестимерном пространстве переменных Эйлера, знаменитый интеграл Ковалевской, который гарантирует интегрируемость; она также смогла обнаружить переменные $s_{1}, s_{2}$ в фазовом пространстве, в которых интегрирование уравнений сводится к задаче обрашения Абеля-Якоби для римановой поверхности рода 2. Отсюда, в частности, следует разрешимость задачи в тета-функциях.

В другой работе того же года [3] Ковалевская показала, что кроме случаев Эйлера, Лагранжа и обнаруженного ею не существует других твердотельных систем, которые были бы аналогичным образом интегрируемы при каждом значении постоянной плошадей. Это дает трем упомянутым системам чрезвычайно специальный статус среди всех систем динамики твердого тела. Однако, в то время как волчки Эйлера и Лагранжа благодаря их простоте всегда были популярны среди физиков, волчок Ковалевской долгое время оставался почти неизвестньм. В классической книге Клейна и Зоммерфельда о волчках [4] он упомянут лишш мимоходом. Причина заключается, по-видимому, в его значительной сложности. Кроме

Работа первого автора выполнена при поддержке гранта Президента РФ (грант № 96-1596868) и Российского фонда фундаментальных исследований (грант № 99-01-01249).

Работа третьего автора выполнена при поддержке программы "Ведущие научные школы" (грант № 96-15-96142) и Российского фонда фундаментальных исследований (грант № 98-0100240).

(C) А. В. БолСинов, П. РихтеР, А. Т. ФОменко 2000 
примечательного исключения в виде ранней аналитической работы Кеттера [5], эта система до сравнительно недавнего времени изучалась в основном русскими математиками. Обзор результатов, полученных в течение прошедших 100 лет, содержится в [6].

Мы упомянем здесь лиш некоторые, наиболее важные на наш взгляд достижения в этом направлении. В первых работах основное внимание уделялось аналитическим свойствам системы. Н.Е. Жуковский [7] изучил в деталях связь между переменньми Ковалевской $\left(s_{1}, s_{2}\right)$ и переменньми Эйлера $\left(p, q, r, \gamma_{1}, \gamma_{2}, \gamma_{3}\right)$. Следующим важным шагом для более глубокого понимания волчка Ковалевской была классификация особых орбит с алгебраической точки зрения, полученная Апшельротом [8]. В целом природа бифуркационной диаграммы, т.е. множества критических значений отображения момента, была исследована Харламовым в [9]. Начиная с этой работы внимание стало перемещаться в сторону топологической структуры фазового пространства системы. Ее описание стало одной из наиболее вызывающих задач для теории топологической классификации интегрируемых систем с двумя степенями свободы, которая была развита А.Т. Фоменко в 80-х годах (см. [10]-[14]). Эти работы привлекли к системе Ковалевской внимание физиков, которьм удалось вычислить переменные действия и дать описание изоэнергетических поверхностей в терминах этих переменных [15]-[17].

Следует безусловно упомянуть также работы [18]-[22], в которых всесторонне проанализирована алгебро-геометрическая структура волчка Ковалевской, найдены его нетривиальные обобшения, представление Лакса и изоморфизмы с другими известными интегрируемыми системами.

Волчок Ковалевской представляет собой систему с тремя степенями свободы, которая благодаря $S^{1}$-симметрии редуцируется к однопараметрическому семейству систем с двумя степенями свободы. В качестве параметра $l$ берется значение углового момента относительно оси гравитации (постоянная площадей). Такова общепринятая точка зрения на ее описание. Оказьвается, что отображение момента $\mathscr{F}_{l}$ из фазового пространства в пространство интегралов $H$ и $K$, где $H$ - интеграл энергии, а $K$ - интеграл Ковалевской, приводит к десяти различным типам слоений Лиувилля на изоэнергетических поверхностях ( $H=$ const) (в предположении, что $l \geqslant 0)$. Структура этих слоений на двумерные торы Лиувилля была описана в терминах так называемых "молекул" в [12], [15]. Однако некоторые из инвариантов, важные для понимания глобальной топологической картины, оставались до настоящего времени неизвестньми. Это - числовые метки $(r, \varepsilon, n)$ на ребрах молекул, которые содержат необходимую информацию о том, как бифуркации, отвечающие вершинам молекулы, взаимодействуют между собой вдоль соединяющих их ребер.

Эта недостающая информация получена в настоящей работе. Топологический анализ случая Ковалевской, таким образом, полностью завершен. Для решения нашей задачи в качестве нового инструмента топологической теории мы развиваем метод круговых молекул. Основная идея состоит в том, чтобы рассмотреть замкнутые кривые, окружающие особые точки бифуркационных диаграмм, и определить допустимые системы координат на семействах торов Лиувилля, ассоциированные с "атомами” соответствующих молекул. Важным является то обстоятельство, что все эти системы координат могут быть заданы в терминах одно- 
значно определенных фундаментальных циклов на торах. На следующем шаге мы определяем взаимное расположение этих циклов и из этой информации получаем так называемые матрицы склейки для молекул, представляющих изоэнергетические поверхности (табл. 4). Хотя матрицы склейки определены неоднозначно, они содержат всю информацию о числовых метках $(r, \varepsilon, n)$. Окончательньй результат представлен в табл. 3 .

Настоящая работа состоит из трех частей. В $\S 2$ мы собрали необходимые идеи и факты из топологической теории интегрируемых гамильтоновых систем. Все обозначения взяты из [13], [23]. В п. 2.1 мы обсу ждаем особые точки бифуркационных диаграмм и показываем, как соответствуюшие им круговые молекулы могут быть использованы в общей ситуации для получения глобальной информации о топологии системы. Кроме того, мы даем списки двух типов круговых молекул: молекулы, относящиеся к невырожденным положениям равновесия (п. 2.2), и молекулы, связанные с вырожденными критическими орбитами (п. 2.3).

Далее, в $\S 3$ мы описываем систему Ковалевской: структуру ее фазового пространства, уравнения движения и различные версии бифуркационных диаграмм. В п. 3.2 мы рассматриваем проекцию трехмерной бифуркационной диаграммы системы в целом, а в п. 3.3 переходим на точку зрения редуцированной системы. Мы также приводим классификацию Аппельрота критических орбит и описываем два типа сингулярных точек (относительные положения равновесия и вырожденные критические орбиты).

В $\S 4$ мы применяем метод круговых молекул для описания глобальной топологии системы Ковалевской. В п. 4.1 описывается полный набор допустимых систем координат для всех бифуркаций; затем в п. 4.2 устанавливается их взаимное расположение. Наконец, в п.п. 4.3 и 4.4 мы используем эту информацию для получения окончательного результата в табл. 3 .

\section{§2. Топология лиувиллевых слоений}

Рассмотрим интегрируемую гамильтонову систему на симплектическом многообразии $\left(M^{4}, \omega\right)$ с гамильтонианом $H$ и дополнительным интегралом $f$. Пусть $\Sigma$ - бифуркационная диаграмма отображения момента $\mathscr{F}$ интегрируемой системы. Диаграмма $\Sigma$ расположена в плоскости $\mathbb{R}^{2}(H, f)$. Будем считать, что $\Sigma$ представляет собой набор гладких кривых $\gamma_{1}, \ldots, \gamma_{k}$, которые могут пересекаться или касаться друг друга в некоторых точках. Пусть при этом каждая гладкая дуга из семейства $\gamma_{1}, \ldots, \gamma_{k}$ удовлетворяет условию боттовости. Другими словами, соответствующие бифуркации имеют эллиптический или гиперболический тип, т.е. в нашей терминологии отвечают некоторым атомам. Отметим, что диаграмма $\Sigma$ может содержать изолированные точки.

В этой вводной части мы напомним некоторые необходимые нам факты из теории лиувиллевых слоений фазовых пространств и их интерпретацию в терминах так называемых молекул. Главное внимание будет уделено особым точкам бифуркационной диаграммы и структуре соответствующих круговых молекул.

2.1. Особые точки бифуркационных диаграмм. Особые точки бифуркационной диаграммы $\Sigma$ - это точки пересечения, касания, излома гладких дуг диаграммы или же изолированные точки, которые этим дугам вообще не принад- 
лежат. Другими словами, мы берем все те точки, в которых $\Sigma$ не является гладкой кривой (см. [23; т. 1, гл. 9]).

Обозначим множество особых точек диаграммы $\Sigma$ через $\Sigma_{0}$. Будем считать, что $\Sigma_{0}$ - это конечное множество точек. Если представлять $\Sigma$ в виде одномерного клеточного комплекса, то $\Sigma_{0}-$ это как раз множество его вершин. Ребра этого комплекса - это в точности дуги $\gamma_{1}, \ldots, \gamma_{k}$.

Напомним, что гладкая кривая $\tau$ без самопересечений в плоскости $\mathbb{R}^{2}(H, f)$ называется допустимой, если она пересекает бифуркационную диаграмму $\Sigma$ трансверсально и не проходит через особые точки $\Sigma$.

Полньй прообраз $Q_{\tau}=\mathscr{F}^{-1}(\tau)$ кривой $\tau$ в $M^{4}$ - это трехмерное гладкое многообразие со структурой лиувиллева слоения, все особенности которого являются боттовскими. Тем самым, на $Q_{\tau}$ возникает инвариант этого слоения - меченая молекула $W^{*}$, которую мы обозначим через $W^{*}(\tau)$. Эта молекула не меняется при гладкой изотопии $\tau$ в классе допустимых кривых.

Пусть теперь $y_{0}$ из $\Sigma_{0}$ - некоторая изолированная особая точка бифуркационной диаграммы. Рассмотрим окружность $\tau$ малого радиуса с центром в точке $y_{0}$. Предположим, что $\tau$ - допустимая кривая и что она остается таковой при уменьшении ее радиуса. Тогда корректно определена молекула $W^{*}(\tau)$.

ОПРЕДЕЛЕНИЕ 1. Молекула $W^{*}(\tau)$ называется круговой молекулой особой точки $y_{0}$ бифуркационной диаграммы $\Sigma$ и обозначается через $W^{*}\left(y_{0}\right)$.

Общая идея использования круговых молекул для описания глобальной структуры лиувиллева слоения на изоэнергетических поверхностях такова. Круговая молекула является локальным инвариантом особенности, поэтому обычно ее бывает вычислить легче, чем молекулу для изоэнергетической поверхности, являющуюся уже глобальным инвариантом. Если тип особенности, отвечающей особой точке $y_{0} \in \Sigma$, понят и описан, то нахождение соответствуюшей круговой молекулы не составляет труда. Более того, в конкретных задачах обычно встречаются лишь особенности из некоторого конечного списка. Поэтому можно заранее составить некоторую таблицу круговых молекул для таких наиболее типичных особенностей. Этой таблицей можно затем пользоваться при исследовании конкретных систем.

Предположим теперь, что мы вычислили все круговые молекулы для всех особых точек данной бифуркационной диаграммы. Тогда меченую молекулу $W^{*}(\tau)$ для любой допустимой кривой на плоскости $\mathbb{R}^{2}(H, f)$ можно склеить из кусочков круговых молекул. Оказывается, эта процедура позволяет многое узнать об искомой молекуле $W^{*}(\tau)$ (в частности, о ее метках). Иногда даже удается полностью вычислить молекулу $W^{*}(\tau)$. Проиллюстрируем эту мысль на рис. 1 . На рис. 1а) показана некоторая бифуркационная диаграмма, у которой отмечены особые точки $y_{1}, y_{2}, y_{3}$. Далее, здесь же изображена некоторая допустимая кривая $\tau=\left\{H=h_{0}\right\}$. Эта кривая пересекает четыре гладких участка $\gamma_{1}, \gamma_{2}, \gamma_{3}, \gamma_{4}$ бифуркационной диаграммы $\Sigma$. Каждый из этих участков $\gamma_{i}$ отвечает некоторому атому $V_{i}$, т.е. невырожденной бифуркации торов Лиувилля, см. [23]. Сразу отметим, что эти атомы те же самые, что и атомы, участвующие в круговых молекулах. Более того, метки $r$ на ребрах между ними тоже совпадают с соответствующими $r$-метками для круговых молекул. Например, молекулы $W^{*}(\tau)$ и $W^{*}\left(y_{1}\right)$ имеют 
общий фрагмент вида $V_{1}-V_{2}$ (рис. 1$)$.

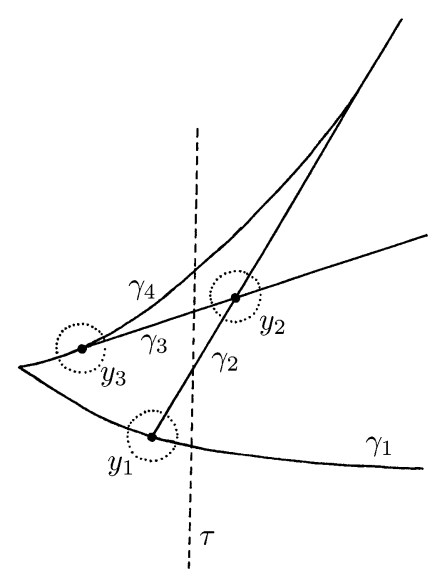

a)

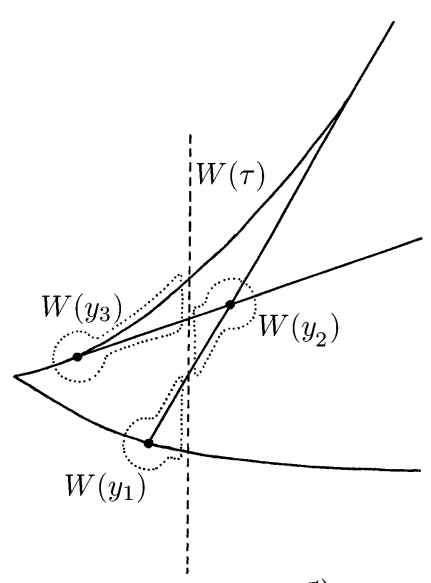

б)

Рис. 1

На рис. 1б) показана изотопия ребер круговых молекул, деформирующая эти ребра в соответствующие отрезки допустимой кривой $\tau$. Из рис. 1б) видно, что при такой изотопии топология слоения Лиувилля не меняется. В результате из фрагментов круговых молекул $W^{*}\left(y_{1}\right), W^{*}\left(y_{2}\right), W^{*}\left(y_{3}\right)$ составляется искомая молекула $W^{*}(\tau)$, включающая информацию о склейке торов Лиувилля.

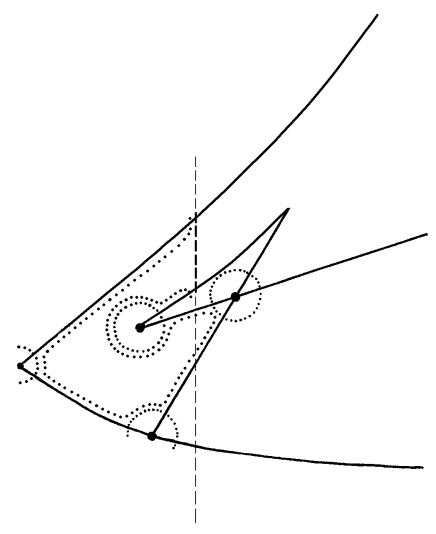

Рис. 2

Описанная схема рассуждений, конечно, условна в том смысле, что конкретная ситуация может оказаться сложнее. Мы описали лишп относительно простой случай. Иногда может случиться так, что одному отрезку кривой $\tau$ отвечает не одно, а несколько ребер круговых молекул особых точек (см. пример на рис. 2). В этом случае требуются дополнительные соображения, чтобы восстановить матрицы склеек для $W^{*}(\tau)$ из соответствующих матриц в круговых молекулах. Мы обсудим эту процедуру ниже. 
2.2. Круговые молекулы невырожденных положений равновесия. Напомним некоторые основные факты о невырожденных особенностях отображения момента. Топология таких особенностей рассматривалась многими авторами. См., например, работы Л.М. Лермана и Я.Л. Уманского [24], В. С. Матвеева [25], Нгуен Тьен Зунга [26]. Рассмотрим изолированное положение равновесия $x \in M^{4}$ интегрируемой гамильтоновой системы $\left(M^{4}, \omega, H, f\right)$. Ее образ, очевидно, является особой точкой бифуркационной диаграммы $\Sigma$. Невырожденность точки $x$ означает, что собственные значения линеаризованной гамильтоновой системы с гамильтонианом $H+c f$ (и некоторым подходящим коэффициентом $c$ ) все различны и отличны от нуля. С точки зрения отображения момента точка $x$ может принадлежать к одному из следующих четырех типов, в зависимости от собственных значений:

1) центр-центр: $i \lambda,-i \lambda, i \mu,-i \mu$;

2) центр-седло: $i \lambda,-i \lambda, \mu,-\mu$;

3) седло-седло: $\lambda,-\lambda, \mu,-\mu$;

4) фокус-фокус: $\lambda+i \mu, \lambda-i \mu,-\lambda+i \mu,-\lambda-i \mu$.

Здесь $\lambda$ и $\mu$-отличные от нуля вещественные числа.

ЗАмечАнИЕ 1. Вместо исходного гамильтониана $H$ мы рассматриваем здесь линейную комбинацию $H$ и $f$, поскольку для самого гамильтониана собственные значения могут совпадать или быть равными нулю. Однако эта проблема может быть устранена взятием подходящей линейной комбинации. Другими словами, точка $x$ может быть вырожденной для гамильтониана $H$, но невырожденной для отображения момента $\mathscr{F}=(H, f)$.

ЗАмЕчАниЕ 2. Не все положения равновесия отображаются в особые точки бифуркационной диаграммы. В качестве примера рассмотрим комбинацию осциллятора с ротатором: $H\left(p_{1}, p_{2}, q_{1}, q_{2}\right)=p_{1}^{2}+q_{1}^{2}+p_{2}^{2}$ и $f=p_{2}$. Здесь положение равновесия не является изолированным, поскольку координата $q_{2}$ пробегает целую окружность (мы считаем, что координата $q_{2}$ определена по модулю $2 \pi$ ). Эта окружность является на самом деле невырожденной одномерной орбитой, порожденной функцией $f$ (которая с точки зрения слоения может быть рассмотрена как независимьй гамильтониан).

Для всех четырех типов невырожденных особенностей бифуркационной диаграммы круговые молекулы полностью описаны в [25], [27]; см. также [23; т. 1, гл. 9]. В то же время представляет интерес посмотреть, какие из этих круговых молекул действительно появляются в конкретных задачах классической механики и математической физики. Мы составили такой список на основе проведенного в последние годы разными авторами обширного анализа конкретных гамильтоновых систем (см. [12], [15], [28]-[30]).

Результат приведен в табл. 1. Для каждой круговой молекулы мы указали те классические случаи интегрируемости, где эта молекула встречается.

Этот список может быть неполным, поскольку мы исследовали пока не все случаи интегрируемости, известные сегодня. Тем не менее, наш список возник в результате обработки большого числа случаев интегрируемости. В этом смысле он достаточно представителен. 
ТАБлицА 1. Круговые молекулы невырожденных положений равновесия

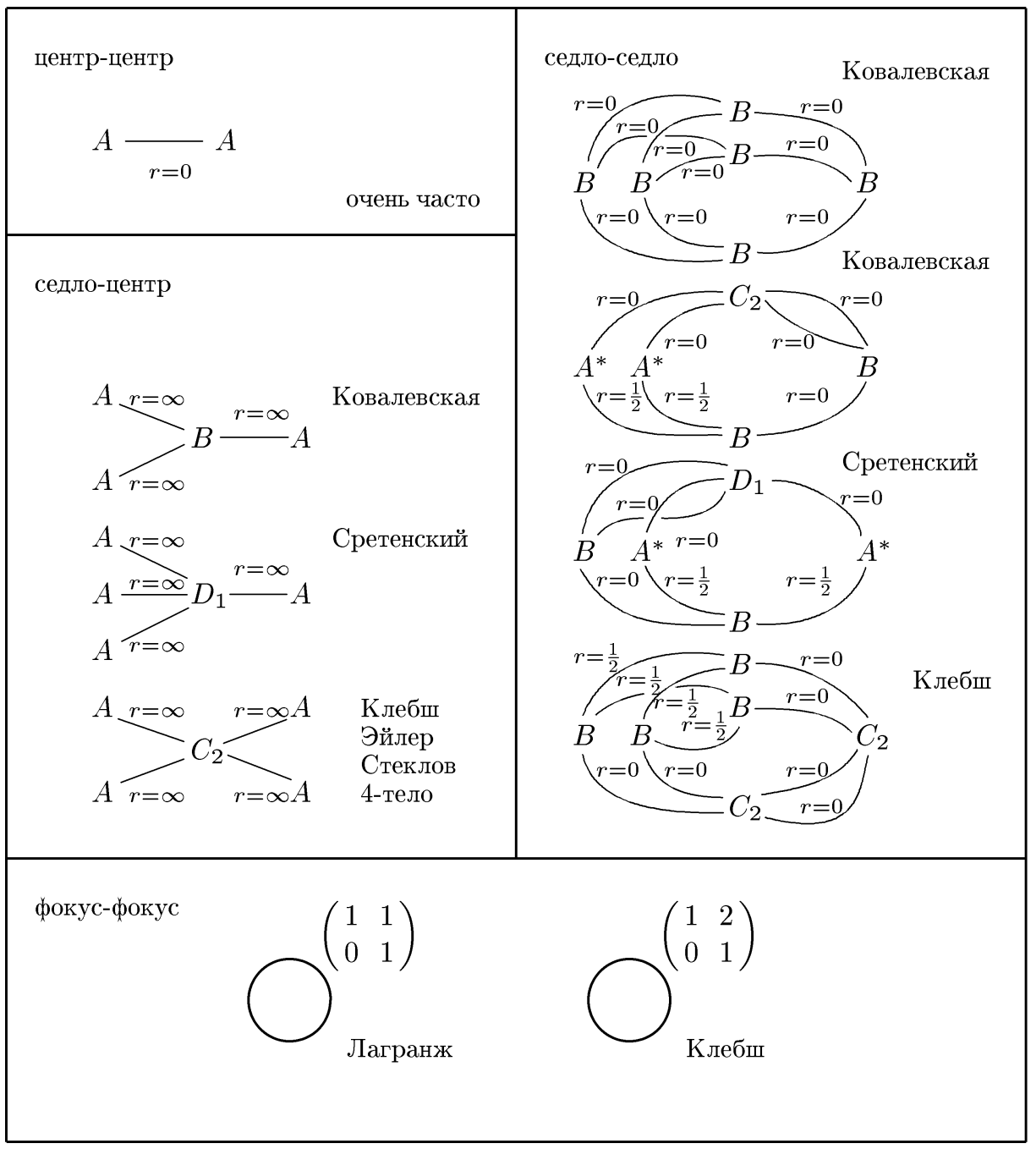

2.3. Круговые молекулы, отвечающие вырожденным одномерным орбитам. Невырожденные одномерные орбиты интегрируемой гамильтоновой системы могут иметь два типа: эллиптический (устойчивый) и гиперболический (неустойчивый). Такие орбиты в случае двух степеней свободы всегда образуют однопараметрические семейства, переходящие при отображении момента в регулярные участки бифуркационной диаграммы $\Sigma$. Особые точки $\Sigma_{0}$ появляются в результате вырождений, когда при изменении некоторых параметров орбита меняет свой тип. В примерах из классической механики это обычно означает один из двух сценариев: а) переход от эллиптической особенности к гиперболической или наоборот (бифуркация "удвоения периода" и "вилка" (pitchfork)); б) рождение или исчезновение особенности (бифуркация "седло-узел”). Во всех случаях переход характеризуется существованием орбиты с нулевьм собственньм значением; такие орбиты называются параболическими. 
Ниже мы обсуждаем наиболее часто встречающиеся особенности и приводим список соответствующих круговых молекул (см. табл. 2).

Пример 1. Бифуркация седло-узел. Рассмотрим в $\mathbb{R}^{3}\left(p_{1}, q_{1}, p_{2}\right)$ две функции: $H=p_{2}$ и $f=f\left(p_{1}, q_{1}, p_{2}\right)$. Линии уровня функции $f$ на горизонтальных плоскостях $p_{2}=$ const изображены на рис. 3 . Видно, что с ростом $p_{2}$ происходит рождение двух невырожденных особых точек разных типов, а именно, эллиптической и гиперболической точек. $\mathrm{B} \mathbb{R}^{3}$ две функции $H$ и $f$ задают одномерное слоение. Из него легко изготовить двумерное слоение на $\mathbb{R}^{3} \times S^{1}$, умножив эту картину прямьм образом на окружность $S^{1}$. Мы будем считать, что на окружности $S^{1}$ задана периодическая координата $q_{2}$, так что в $\mathbb{R}^{3} \times S^{1}$ мы получаем все четыре симплектические координаты $p_{1}, q_{1}, p_{2}, q_{2}$. Ясно, что функции $H(p, q)$ и $f(p, q)$ коммутируют и задают слоение Лиувилля в 4-мерном симплектическом многообразии $\mathbb{R}^{3} \times S^{1}$. Пространство $\mathbb{R}^{3}\left(p_{1}, q_{1}, p_{2}\right)$ при этом может рассматриваться как сечение.

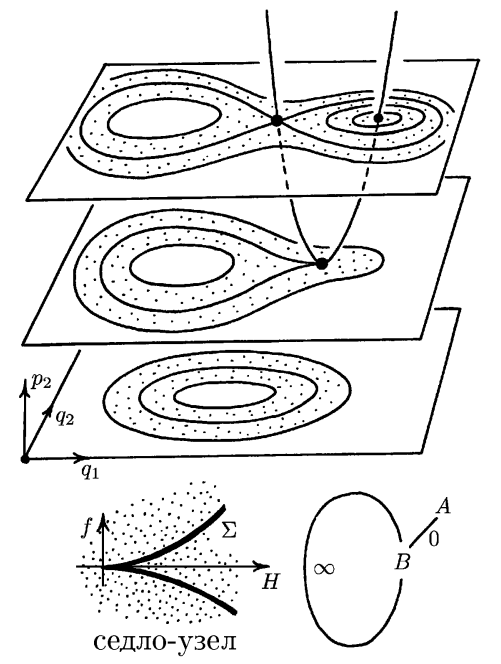

Рис. 3

Соответствующая этой интегрируемой системе бифуркационная диаграмма отображения момента $\mathscr{F}: \mathbb{R}^{3} \times S^{1} \rightarrow \mathbb{R}^{2}(H, f)$ показана на рис. 3 . Видно, что это кривая с точкой возврата, целиком содержащаяся в образе отображения момента $\mathscr{F}$. Соответствуюшая круговая молекула $W_{\tau}$, полученная при обходе точки возврата, также показана на рис. 3 .

В качестве функции $f$ можно взять функцию, имеющую в окрестности начала координат (где и расположена особенность) следующий вид:

$$
f=p_{1}^{2}+q_{1}^{3}-p_{2} q_{1}
$$

Это в точности локальный канонический вид функции $f$ в случае параболической особенности [23], [31]. В этом случае множество особых точек отображения, задаваемого парой функций

$$
\left(H=p_{2}, \quad f=p_{1}^{2}+q_{1}^{3}-p_{2} q_{1}\right),
$$


представляет собой параболу в $\mathbb{R}^{3}$, лежащую в плоскости $p_{1}=0$ и задаваемую уравнением $p_{2}=3 q_{1}^{2}$. При $q_{1}>0$ получаем особые точки эллиптического типа, при $q_{1}<0$ - гиперболического типа, а при $q_{1}=0$ получаем параболическую особую точку. Бифуркационная диаграмма $\Sigma$ для отображения момента $\mathscr{F}$ в плоскости $(H, f)$ получается подстановкой $p_{1}=0, q_{1}^{2}=p_{2} / 3=H / 3$ в уравнение для $f$ :

$$
f=-2 q_{1}^{3}= \pm \frac{2}{3 \sqrt{3}} H^{3 / 2} .
$$

В случае, когда интегрируемая система удовлетворяет дополнительным условиям, например, допускает $\mathbb{Z}_{2}$-симметрию, на особом слое могут одновременно появиться две параболические одномерные орбиты. Такой случай изображен на рис. 4. Здесь же показана соответствующая круговая молекула.

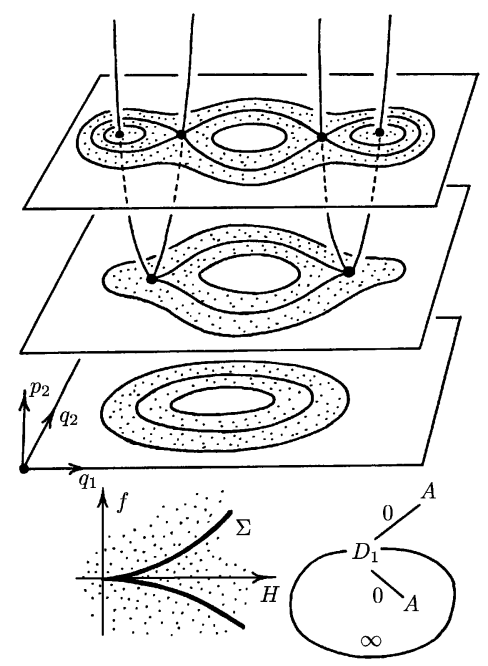

Рис. 4

ПРИмЕР 2. Эллиптическая вилка (pitchfork) и эллиптическая бифуркация удвоения периода. Для описания качественной картины снова рассмотрим в $\mathbb{R}^{3}\left(p_{1}, q_{1}, p_{2}\right)$ две функции: $H=p_{2}$ и $f=f\left(p_{1}, q_{1}, p_{2}\right)$. Линии уровня функции $f$ на горизонтальных плоскостях $p_{2}=$ const изображены на рис. 5 . Видно, что с ростом $H$ происходит превращение эллиптической особенности в три невырожденные особые точки разных типов, а именно, две эллиптических точки и одна гиперболическая. $\mathrm{B} \mathbb{R}^{3}$ две функции $H$ и $f$ задают одномерное слоение. Из него снова легко изготовить двумерное слоение на $\mathbb{R}^{3} \times S^{1}$. Однако здесь, в отличие от параболического случая, имеются две возможности благодаря $\mathbb{Z}_{2}$-симметрии.

Первый способ: нужно умножить эту картину прямым образом на окружность $S^{1}$. Этот случай условно назовем ориентируемым. Здесь мы будем считать, что на окружности $S^{1}$ задана $2 \pi$-периодическая координата $q_{2}$, так что в $\mathbb{R}^{3} \times S^{1}$ мы получаем все четыре симплектические координаты $p_{1}, q_{1}, p_{2}, q_{2}$. Ясно, что функции 


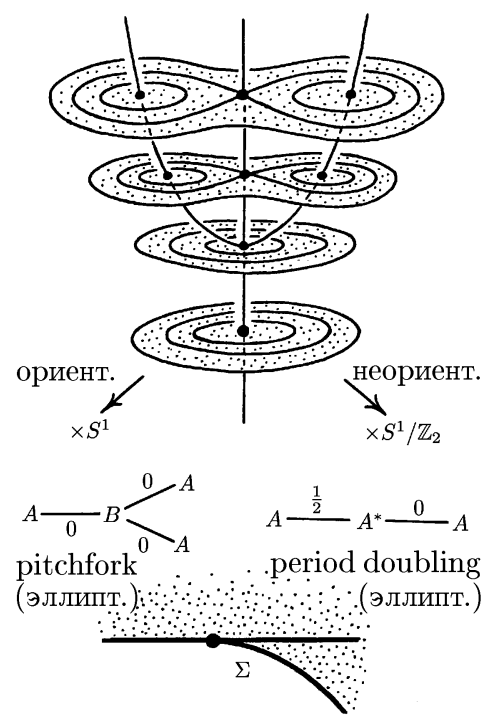

Рис. 5

$H(p, q)$ и $f(p, q)$ коммутируют и задают слоение Лиувилля в 4-мерном симплектическом многообразии $\mathbb{R}^{3} \times S^{1}$. На данной изоэнергетической поверхности с достаточно большим значением $H$ расположены две критические орбиты эллиптического типа, связанные друг с другом симметрией. Переход от одного к трем критическим орбитам (одномерным торам) - это и есть бифуркация "вилка" (pitchfork).

Второй способ, который мы назовем неориентируемым, состоит в следующем. Нужно умножить описанную в $\mathbb{R}^{3}$ картину на окружность, после чего профакторизовать по действию группы $\mathbb{Z}_{2}$. При этом действие образуюшей группы $\mathbb{Z}_{2}$ на $\mathbb{R}^{3} \times S^{1}$ задается так:

$$
\left(p_{1}, q_{1}, p_{2}, q_{2}\right) \longrightarrow\left(-p_{1},-q_{1}, p_{2}, q_{2}+\pi\right) .
$$

Тот же результат можно получить, умножив картину, показанную на рис. 5 , на $q_{2}$-отрезок $[0, \pi]$ и склеив затем основания $q_{2}=0$ и $q_{2}=\pi$ получившегося цилиндра по повороту на угол $\pi$ вокруг вертикальной оси $p_{2}$. В результате мы приходим к бифуркации удвоения периода.

Соответствующая этой интегрируемой системе бифуркационная диаграмма отображения момента $\mathscr{F}: \mathbb{R}^{3} \times S^{1} \rightarrow \mathbb{R}^{2}(H, f)$ показана на рис. 5. Две соответствуюшие круговые молекулы $W_{\tau}^{\text {op }}$ и $W_{\tau}^{\text {неор }}$, получающиеся при обходе вокруг особой точки, также показаны на рис. 5 .

В качестве функции $f$ можно взять, например, функцию, локально имеющую следуюший вид:

$$
f=p_{1}^{2}+q_{1}^{4}-p_{2} q_{1}^{2} .
$$

Множество особых точек задается в $\mathbb{R}^{3}$ уравнениями:

$$
\begin{aligned}
q_{1}=0, & p_{1}=0 \quad \text { (вертикальная прямая) }, \\
2 q_{1}^{2}=p_{2}, & p_{1}=0 \quad \text { (парабола в вертикальной плоскости). }
\end{aligned}
$$


Бифуркационная диаграмма $\Sigma$ для отображения момента $\mathscr{F}$ в плоскости $(H, f)$ состоит из двух кусков. Первый кусок - это прямая $f=0$. Второй кусок - это половина параболы $f=-H^{2} / 2, H>0$ (рис. 5). Отметим, что образ отображения момента содержится в области, заштрихованной на рис. 5. Круговая молекула в результате не содержит циклов, поскольку мы не можем совершить полного обхода вокруг особой точки, не покидая образа отображения момента.

Пример 3. Гиперболическая вилка (pitchfork) и гиперболическая бифуркация удвоения периода. Этот случай аналогичен предыдущему, но здесь нужно поменять местами эллиптические и гиперболические особенности. В результате получится картина в $\mathbb{R}^{3}$, изображенная на рис. 6 . Здесь одна гиперболическая особенность преврашается в одну эллиптическую и две гиперболические. Мы снова различаем два случая: ориентируемый и неориентируемый.

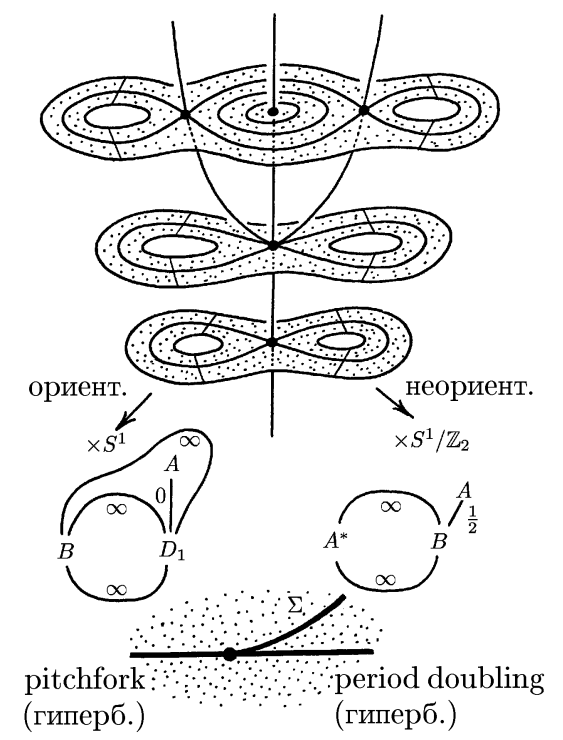

Рис. 6

Как и в примере 1 , мы полагаем $H=p_{2}$, а в качестве функции $f$ можно взять, например, функцию, локально имеющую следующий вид:

$$
f=p_{1}^{2}-q_{1}^{4}+p_{2} q_{1}^{2}
$$

Множество особых точек задается в $\mathbb{R}^{3}$ уравнениями:

$$
\begin{aligned}
q_{1}=0, & p_{1}=0 \quad \text { (вертикальная прямая) }, \\
2 q_{1}^{2}=p_{2}, & p_{1}=0 \quad \text { (парабола в вертикальной плоскости). }
\end{aligned}
$$

Бифуркационная диаграмма $\Sigma$ для отображения момента $\mathscr{F}$ в плоскости $(H, f)$ аналогична предыдущему случаю и состоит из двух кусков. Первый кусок - это прямая $f=0$. Второй кусок - это половина параболы $f=H^{2} / 2, H>0$ (рис. 6). Отметим, впрочем, что теперь образу отображения момента принадлежит целая окрестность точки $(H, f)=(0,0)$. Это приводит к иному характеру круговой молекулы, которая теперь содержит цикл. 
ПримеР 4. Модифишированные гиперболическая вилка (pitchfork) и гиперболическая бифуркация удвоения периода. Локально этот случай совпадает с предыдущим примером. Так же, как в примере 3 , устроены здесь функции $H$ и $f$, бифуркационная диаграмма $\Sigma$, множество критических точек отображения момента. Однако глобальная картина все же отличается. Соединяя по-другому сепаратрисы, мы получаем в сечении вместо атома $D_{1}$ атом $C_{2}$ (рис. 7 ). Получающиеся здесь две круговые молекулы, в ориентируемом и неориентируемом случаях, показаны на рис. 7.

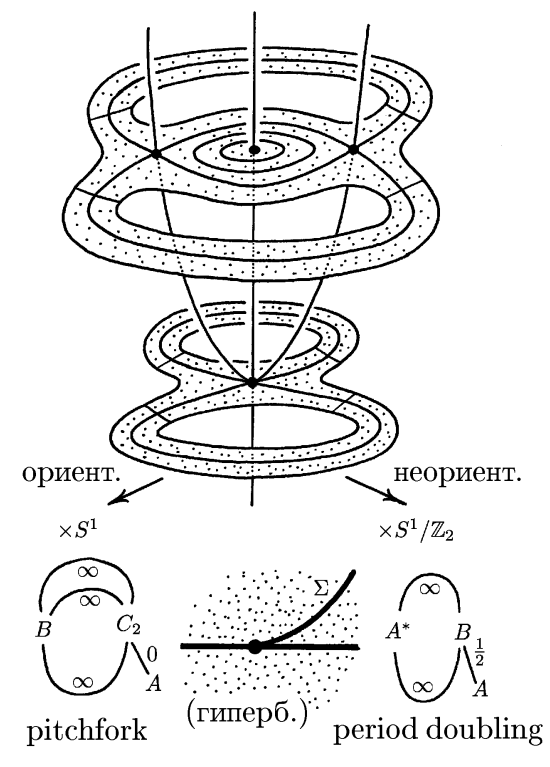

РИс. 7

Стоит обратить внимание, что в неориентируемом случае круговая молекула получается такой же, как и в примере 3. Это не случайно. Дело в том, что соответствующие два слоения Лиувилля диффеоморфны (хотя это не очевидно).

В заключение мы приводим табл. 2, в которой собраны перечисленные выше типичные круговые молекулы особых точек бифуркационных диаграмм. Здесь же указаны некоторые известные случаи интегрируемости, где такие круговые молекулы появляются.

Мы ограничились здесь рассмотрением только некоторых примеров вырожденных особенностей. Среди них можно выделить класс так называемых устойчивых особенностей, сохраняющих свой топологический тип при возмущении системы в классе интегрируемых систем. Классификация таких особенностей получена В.В. Калашниковьм (мл.) [31]. Там же указаны и круговые молекулы для всех таких особенностей. В табл. 2 устойчивыми являются особенности с номерами 1, 4, 6 . Появление в реальных примерах неустойчивых особенностей с номерами 2, 3, 5 не должно нас удивлять. Это объясняется наличием дополнительной $\mathbb{Z}_{2}$-симметрии, которая препятствует их разрушению. 
ТАБлицА 2. Круговые молекулы вырожденных одномерных орбит

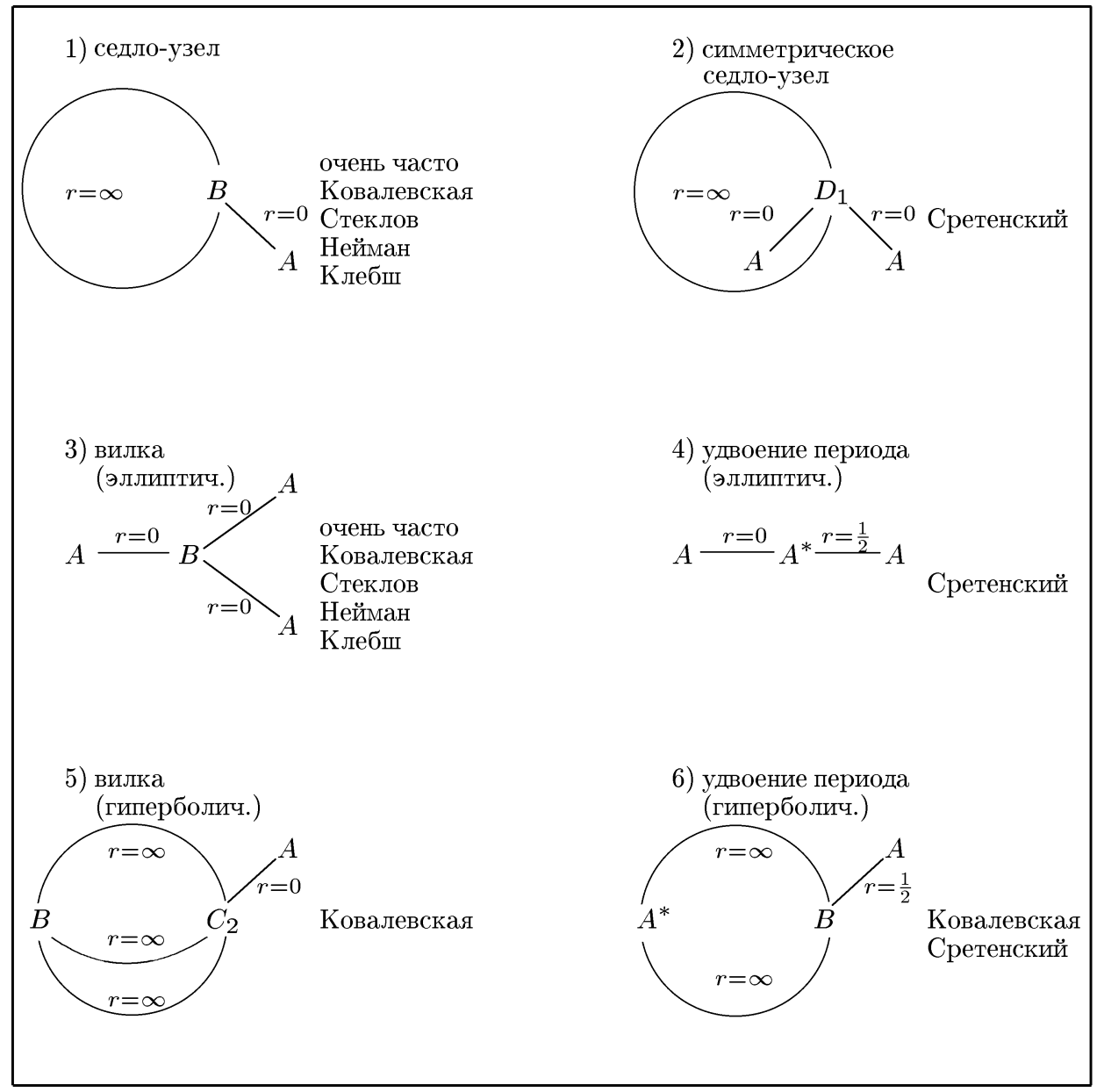

\section{§3. Волчок Ковалевской}

Твердое тело с закрепленной точкой в постоянном гравитационном поле имеет конфигурационное пространство, диффеоморфное группе $\mathrm{SO}(3)$, и допускает $S^{1}$-симметрию, а именно $\varphi$-вращение вокруг вектора гравитационного поля (его направление мы будем в дальнейшем называть вертикальньм). Эта система имеет три степени свободы, но после редукции по указанной симметрии она может рассматриваться как система с двумя степенями свободы, конфигурационным пространством которой служит сфера Пуассона. Удобно вложить эту сферу в пространство $\mathbb{R}^{3}(\gamma)$ и рассматривать шестимерное пространство $\mathbb{R}^{6}(\boldsymbol{\gamma}, \boldsymbol{l})$ в качестве фазового пространства, где $\boldsymbol{l}=\left(l_{1}, l_{2}, l_{3}\right)$ - угловой момент и $\gamma=\left(\gamma_{1}, \gamma_{2}, \gamma_{3}\right)$ - единичньй вертикальный вектор. Оба вектора записаны в системе координат, жестко связанной с телом, оси которой направлены по главным осям инерции. Это фазовое пространство имеет естественную пуассонову структуру

$$
\left\{\gamma_{i}, \gamma_{j}\right\}=0, \quad\left\{l_{i}, \gamma_{j}\right\}=\varepsilon_{i j k} \gamma_{k}, \quad\left\{l_{i}, l_{j}\right\}=-\varepsilon_{i j k} l_{k} .
$$


Эта же структура может быть записана в компактной форме в виде матрицы

$$
\boldsymbol{P}=\left(\begin{array}{cc}
0 & \widehat{\gamma} \\
-\widehat{\gamma} & \widehat{l}
\end{array}\right)
$$

где $\widehat{\boldsymbol{\gamma}}$ и $\widehat{\boldsymbol{l}}$ - кососимметрические $(3 \times 3)$-матрицы, отвечающие векторам $\boldsymbol{\gamma}, \boldsymbol{l}$ соответственно, $\widehat{\gamma}_{i j}=-\varepsilon_{i j k} \gamma_{k}$ и $\widehat{l}_{i j}=-\varepsilon_{i j k} l_{k}$. Эта структура имеет две функции Казимира:

$$
\begin{array}{ll}
C=\gamma_{1}^{2}+\gamma_{2}^{2}+\gamma_{3}^{2} & \text { (геометрический интеграл), } \\
L=\gamma_{1} l_{1}+\gamma_{2} l_{2}+\gamma_{3} l_{3} & \text { (угловой момент, или постоянная площадей). }
\end{array}
$$

Эти функции являются первыми интегралами любого гамильтонова движения (т.е. $\boldsymbol{P} \nabla C \equiv 0$ и $\boldsymbol{P} \nabla L \equiv 0)$. Это в точности отражает тот факт, что наша система имеет лиш две эффективных степени свободы. Физические значения для $C$ и $L$ суть $C=1$ и $L=l$ (вертикальная компонента вектора углового момента). Многообразие

$$
M_{l}^{4}=\left\{(\gamma, l) \in \mathbb{R}^{6}: C=1, L=l\right\}
$$

является симплектическим и назьвается редуцированным фазовым пространством. В отличие от полного фазового пространства $T^{*} \mathrm{SO}(3)$, оно игнорирует $\varphi$-движение вокруг вертикальной оси в физическом пространстве. Если мы интересуемся этим движением, оно может быть получено из движения на $M_{l}^{4}$ интегрированием уравнения

$$
\dot{\varphi}=\frac{1}{\gamma_{1}^{2}+\gamma_{2}^{2}}\left(\frac{\gamma_{1} l_{1}}{A_{1}}+\frac{\gamma_{2} l_{2}}{A_{2}}\right),
$$

где $A_{i}$ - главные моменты инерции.

3.1. Уравнения движения и интегралы. Волчок Ковалевской характеризуется тем, что его главные моменты инерции имеют вид $A_{1}=A_{2}=2 A_{3}=2$, а центр масс $\boldsymbol{c}$ расположен в плоскости симметрии эллипсоида инерции, перпендикулярной третьей оси инерции. Без ограничения общности мы можем считать, что $\boldsymbol{c}=(-1,0,0)$. Тогда гамильтониан принимает вид

$$
H=\frac{1}{4}\left(l_{1}^{2}+l_{2}^{2}+2 l_{3}^{2}\right)-\gamma_{1}
$$

Соответствующие уравнения движения (уравнения Эйлера-Пуассона-Ковалевской) принимают вид

$$
\left(\begin{array}{c}
\dot{\gamma} \\
\dot{l}
\end{array}\right)=\boldsymbol{P} \nabla H
$$

или в явном виде

$$
\left(\begin{array}{c}
\dot{\gamma}_{1} \\
\dot{\gamma}_{2} \\
\dot{\gamma}_{3}
\end{array}\right)=\left(\begin{array}{c}
\gamma_{2} l_{3}-\frac{1}{2} \gamma_{3} l_{2} \\
\frac{1}{2} \gamma_{3} l_{1}-\gamma_{1} l_{3} \\
\frac{1}{2} \gamma_{1} l_{2}-\frac{1}{2} \gamma_{2} l_{1}
\end{array}\right) \text { и }\left(\begin{array}{l}
i_{1} \\
i_{2} \\
i_{3}
\end{array}\right)=\left(\begin{array}{c}
\frac{1}{2} l_{2} l_{3} \\
-\frac{1}{2} l_{3} l_{1}-\gamma_{3} \\
\gamma_{2}
\end{array}\right) \text {. }
$$


Кроме двух функций Казимира и интеграла энергии $H=h$ эта система допускает дополнительный интеграл Ковалевской (см. [1]):

$$
K=\left(\frac{l_{1}^{2}-l_{2}^{2}}{4}+\gamma_{1}\right)^{2}+\left(\frac{l_{1} l_{2}}{2}+\gamma_{2}\right)^{2}
$$

Прямая проверка показывает, что $\{H, K\}=0$. Отсюда следует, что система является интегрируемой. Значения интеграла $K$ мы будем обозначать через $k$.

Если волчок Ковалевской рассматривается как система с тремя степенями свободы, то фазовое пространство $T^{*} \mathrm{SO}(3)$ расслоено на трехмерные торы Лиувилля, соответствующие фиксированным значениям первых интегралов $(h, k, l)$. Отображение момента

$$
\mathscr{F}=(H, K, L): T^{*} \mathrm{SO}(3) \longrightarrow \mathbb{R}^{3}(h, k, l)
$$

имеет пять трехмерных областей регулярности [9], [28], отделенных друг от друга двумерными поверхностями, образующими бифуркационную диаграмму $\Sigma_{h, k, l}$, т.е. образ множества точек, в которых ранг дифференциала $d \mathscr{F}$ меньше трех. Система поверхностей $\Sigma_{h, k, l}$ была разделена на четыре класса в пионерской работе Аппельрота [8]. Позже в п. 3.3 мы обсудим их более детально, сместив, однако, акценты с исходной аналитической картины на топологическую точку зрения. Внутри $\Sigma_{h, k, l}$ можно выделить два специальных подмножества: а) одномерное множество особых точек $\Sigma_{h, k, l}^{1}$ (одномерный остов) и б) множество $\Sigma_{h, k, l}^{0}$ изолированных точек, в которых встречаются две или более сингулярных линии. Это последнее множество состоит лишь из семи точек $(h, k, l)$, которые мы будем называть суперсингулярными:

$$
\Sigma_{h, k, l}^{0}=\left\{(2,0, \pm 2),\left(\sqrt{3}, 0, \pm \frac{4}{3} \sqrt[4]{3}\right),\left(\frac{3}{2}, \frac{1}{4}, \pm \sqrt{2}\right),(0,1,0)\right\}
$$

Природа этих точек станет ясна ниже.

Довольно затруднительно представить трехмерную бифуркационную диаграмму $\Sigma_{h, k, l}$ всю целиком. Поэтому в литературе изучаются несколько двумерных версий: проекция $\Sigma_{h, l}$ бифуркационной диаграммы $\Sigma_{h, k, l}$ на плоскость $\mathbb{R}^{2}(h, l)$, сечение $\Sigma_{h, k}^{l} \subset \mathbb{R}^{2}(h, k)$ диаграммы $\Sigma_{h, k, l}$ при фиксированном $l$, сечение $\Sigma_{k, l}^{h} \subset$ $\mathbb{R}^{2}(k, l)$ диаграммы $\Sigma_{h, k, l}$ при фиксированном $h$. Две первые версии обсуждаются в двух следующих пунктах, третья - в [32].

3.2. Бифуркационная диаграмма $\Sigma_{h, l}$. Бифуркационная диаграмма $\Sigma_{h, l}$ рассматривалась в целой серии работ [12], [15], [16], [27], см. также [23]. Схематически она показана на рис. 8; если бы она была показана в реальном масштабе, то точки возврата и области I, J были бы трудно различимы. Кривые этой бифуркационной диаграммы являются проекциями особого множества $\Sigma_{h, k, l}^{1}$. Мы используем линии двух типов, чтобы различить два типа сингулярного поведения. Жирные сплошные линии указывают на бифуркации топологического типа изоэнергетической поверхности, пунктирные линии отвечают за бифуркации лиувиллева слоения при неизменном топологическом типе изоэнергетической поверхности. 


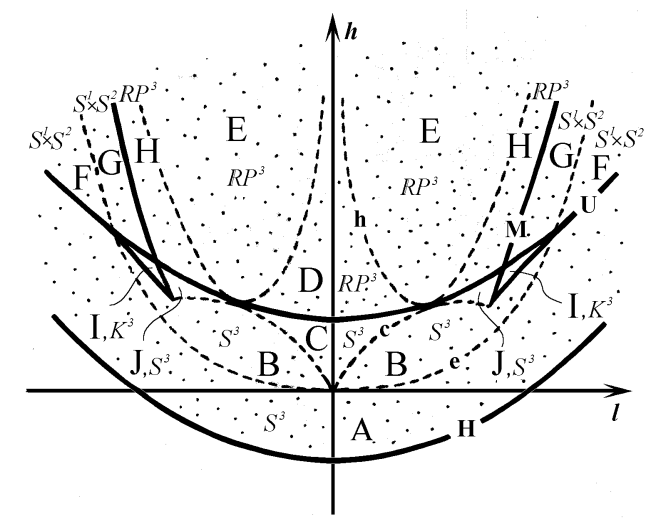

Рис. 8

Под изоэнергетической поверхностью мы понимаем здесь трехмерные подмножества редуцированного фазового пространства вида

$$
Q_{h, l}^{3}=\left\{(\gamma, l) \in M_{l}^{4}: H=h\right\} .
$$

В зависимости от $(h, l)$ сушествует четыре топологических типа $Q_{h, l}^{3}($ не считая пустого множества при $\left.h<\frac{l^{2}}{4}-1\right)$. Как указано на рис. 8, это трехмерная сфера $S^{3}$ при малых значениях энергии или углового момента; трехмерное проективное пространство $\mathbb{R} P^{3}$ при больших $h$ и малых $|l| ; S^{2} \times S^{1}$ при больших $h$ и средних $|l|$; и наконец, многообразие $K^{3}$, являюшееся связной суммой двух экземпляров $S^{2} \times S^{1}$, в малых областях І около $(h,|l|)=(2,2)$. Области, отвечающие различным топологическим типам изоэнергетических поверхностей, разделяются сплошными линиями диаграммы $\Sigma_{h, l}$, которые отвечают относительньм положениям равновесия системы, т.е. решениям уравнения Эйлера-Пуассона-Ковалевской, для которых $\gamma=$ const, $\boldsymbol{l}=$ const:

$\mathrm{H}$ - спящий волчок в подвешенном положении, $\gamma=(1,0,0), l=(l, 0,0)$,

$$
h=\frac{l^{2}}{4}-1, \quad k=(h+2)^{2} ;
$$

$\mathrm{U}$ - спящий волчок, стоящий вертикально, $\gamma=(-1,0,0), \boldsymbol{l}=(-l, 0,0)$,

$$
h=\frac{l^{2}}{4}+1, \quad k=(h-2)^{2} ;
$$

$\mathrm{M}$ - карусельное движение с угловой скоростью $p=l_{1} / 2$ в качестве параметра, $p^{2} \leqslant 1, \gamma=\left(-p^{2}, 0, \sqrt{1-p^{4}}\right)$ и $\boldsymbol{l}=\left(2 p, 0,-\sqrt{1-p^{4}} / p\right)$,

$$
l=-p^{3}-\frac{1}{p}, \quad h=\frac{3}{2} p^{2}+\frac{1}{2 p^{2}}, \quad k^{2}=0 .
$$

В полной системе с тремя степенями свободы относительные положения равновесия демонстрируют только $\varphi$-движения с постоянной скоростью $\dot{\varphi}$, определяемой уравнением, приведенным выше. 
Кривые относительных положений равновесия определяют две пары суперсингулярных точек из $\Sigma_{h, k, l}^{0}$ :

$S_{1}$ - точка касания линий $\mathrm{U}$ и $\mathrm{M}$,

$$
(h, k, l)=(2,0, \pm 2)
$$

$S_{2}$ - точка возврата линии М при

$$
(h, k, l)=\left(\sqrt{3}, 0, \pm \frac{4}{3} \sqrt[4]{3}\right) \approx(1.7320,0, \pm 3.0792)
$$

Пересечение кривых U и М в точках

$$
(h, l)=(2 \sqrt{2}-1, \pm 2 \sqrt{\sqrt{2}-1}) \approx(1.8284,3.3137)
$$

не отвечает никаким точкам из $\Sigma_{h, k, l}^{0}$, поскольку значения $k$ на этих двух кривых различны.

Из поведения кривой М, рассмотренной отдельно, также неясно, является ли точка возврата суперсингулярной точкой: появление точки возврата может быть результатом проекции. Но в дополнение к сплошньп линиям мы имеем пунктирные линии, указывающие на вырождения критических одномерных орбит; в точке возврата кривая с (см. ниже) отходит от кривой М. Поэтому данная точка попадает в $\Sigma_{h, k, l}^{0}$.

Рассмотрим теперь три типа пунктирных линий. Они могут быть охарактеризованы в терминах классификационной схемы, предложенной Аппельротом [8], [16], [32]:

е - вырождение класса Аппельрота I, переход между классами II и III,

$$
h=\frac{1}{2} l^{2}, \quad k=0
$$

c - вырождение класса Аппельрота IV,

$$
h=\frac{3}{2}\left(\frac{l^{2}}{2}\right)^{1 / 3}, \quad k=1-\frac{h^{2}}{3} \quad\left(l^{2} \leqslant \frac{16}{9} \sqrt{3}\right)
$$

h - вырождение класса Аппельрота III, переход внутри класса IV,

$$
h=\frac{l^{2}}{2}+\frac{1}{l^{2}}, \quad k=\frac{1}{l^{4}} .
$$

Эти линии определяют еще две суперсингулярные точки из $\Sigma_{h, k, l}^{0}$ : $S_{3}$ - точка касания линий $\mathrm{U}$, с и h,

$$
(h, k, l)=\left(\frac{3}{2}, \frac{1}{4}, \pm \sqrt{2}\right)
$$

$S_{0}$ - точка касания двух линий с (с разными знаками $l$ ),

$$
(h, k, l)=(0,1,0)
$$


Система кривых диаграммы $\Sigma_{h, l}$ делит плоскость $\mathbb{R}^{2}(h, l)$ на 17 различных областей, отвечающих различным типам лиувиллевых слоений. Если мы отождествим области, отличающиеся только знаком $l$, то останется лишь 10 различных областей, обозначенных на рис. 8 через A, B, . . J. (Отметим, что линия $l=0$ не является особой, поэтому области A, C, D участвуют только один раз.) Соответствующие молекулы были описаны в [12], [15], [16], [29] и в настоящее время хорошо известны (см. табл. 3). Однако до настоящего времени оставалась до конца не известной структура матриц склейки для этих молекул, или, что то же самое, числовые метки $r, \varepsilon, n$ в терминологии [13], [23]. Это в точности тот вопрос, который решается в настояшей работе; метод круговых молекул используется для того, чтобы получить недостающую информацию.

ТАБлицА 3. Меченые молекулы случая Ковалевской

\begin{tabular}{|c|c|c|}
\hline $\begin{array}{l}r=0 \\
\varepsilon=1 \\
A\end{array}$ & 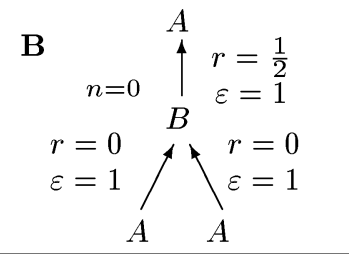 & 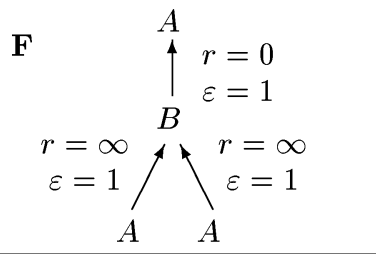 \\
\hline 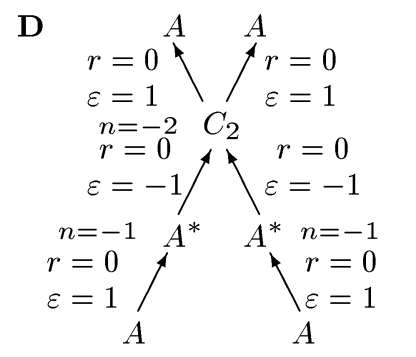 & 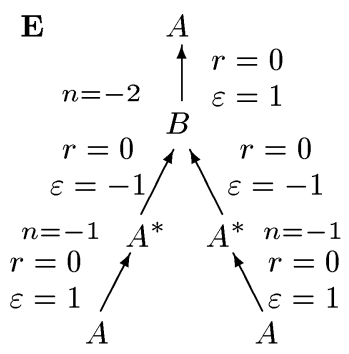 & 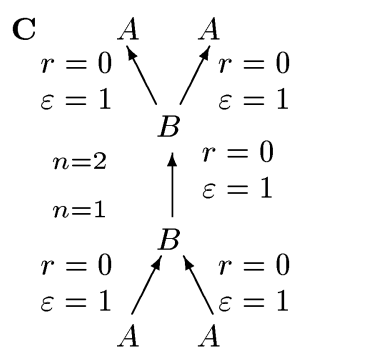 \\
\hline 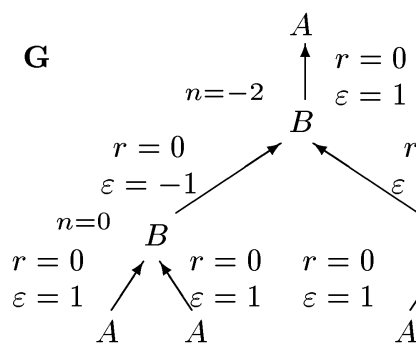 & 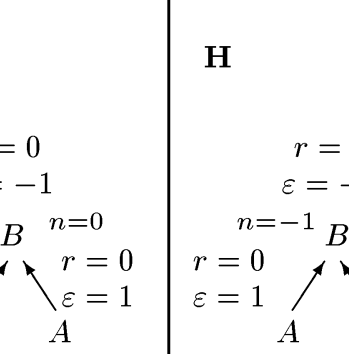 & 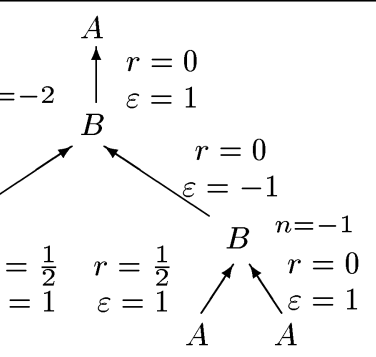 \\
\hline 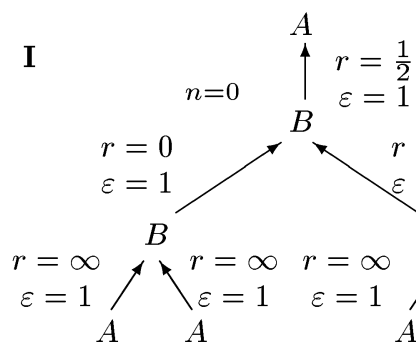 & $\begin{array}{r}\mathbf{J} \\
r=0 \\
\varepsilon=1 \\
r=\infty \\
\varepsilon=1\end{array}$ & 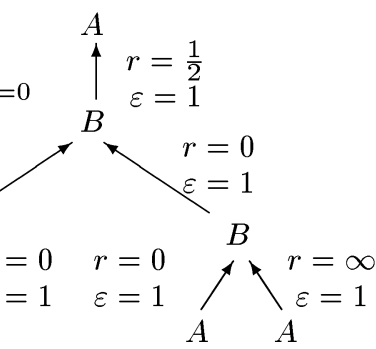 \\
\hline
\end{tabular}


3.3. Бифуркационные диаграммы $\Sigma_{h, k}^{l}$. В дальнейшем мы будем рассматривать волчок Ковалевской как однопараметрическое семейство систем с двумя степенями свободы с параметром $l$ и фазовым пространством $M_{l}^{4}$. Торы Лиувилля для данных значений $(h, l)$ двумерны и лежат в трехмерной изоэнергетической поверхности $Q_{h, l}^{3}$. Отображение момента имеет вид

$$
\mathscr{F}_{l}=(H, K): M_{l}^{4} \rightarrow \mathbb{R}^{2}(h, k),
$$

множество его критических значений является бифуркационной диаграммой $\Sigma_{h, k}^{l}$. Ясно, что это сечение полной диаграммы $\Sigma_{h, k, l}$ двумерной плоскостью $l=$ const.

$\mathrm{K}$ изоэнергетическим поверхностям $Q_{h, l}^{3}$ можно теперь применить обшую теорию лиувиллевой классификации [6], [13], [23].

Бифуркационные диаграммы $\Sigma_{h, k}^{l}$ были впервые построены М.П. Харламовым [9], [28], см. также работы А.А. Ошемкова [12] и М. Одэн [29]. В зависимости от значения $l$ следует различать пять случаев:

a) $l=0$,

б) $0<|l|<\sqrt{2}$,

в) $\sqrt{2}<|l|<\frac{4}{3} \sqrt[4]{3}$

г) $\frac{4}{3} \sqrt[4]{3}<|l|<2$

д) $|l|>2$.

Соответствуюшие бифуркационные диаграммы показаны на рис. 9а)-д). Все эти диаграммы являются точными в том смысле, что соответствуют настоящим аналитическим формулам, приведенным ниже. Все они изображены так, что единица по оси $h$ в два раза больше, чем по оси $k$. Отдельные фрагменты бифуркационных диаграмм, дополнительно изображенные на рис. 9в)-д), увеличены в 40 раз по оси $k$ и в 7 раз по оси $h$.

Для удобства описания возникающих здесь перестроек мы изобразили те же самые диаграммы на рис. 10 и 11a)-д). Качественный вид диаграмм на рис. 10, 11 тот же самьй, что и на рис. 9 , однако некоторые их фрагменты увеличены, чтобы можно было отразить на них всю необходимую нам информацию.

Разные кривые этих бифуркационных диаграмм соответствуют критическим (одномерным) торам, отвечающим четырем классам Аппельрота I, II, III, IV. Множество особых точек $\Sigma_{0}^{l}$ этих диаграмм является пересечением кривых из $\Sigma_{h, k, l}^{1} \mathrm{c}$ плоскостями $l=$ const.

Опишем сначала кривые, отвечающие критическим (одномерньм) торам, т.е. классам Аппельрота. На рис. 10 они обозначены римскими цифрами от I до IV, а на рис. 11 дано более подробное описание с использованием 14 различных меток $\alpha_{i}, \beta_{i}, \gamma_{i}, \delta_{i}$. Мы всюду предполагаем, что $l \geqslant 0$.

Класс I (называемый также классом Делоне): луч $k=0$ c $h \geqslant \frac{l^{2}}{2}$; участки $\delta_{1}$ и $\delta_{2}$ на рис. $11 ;$

Класс ІІ: часть параболы $k=\left(h-\frac{l^{2}}{2}\right)^{2}$, выделяемая условием $\frac{l^{2}}{4}-1 \leqslant h \leqslant \frac{l^{2}}{2}$; участки $\alpha_{1}$ и $\alpha_{2}$ на рис. 11 ;

Класс III: часть той же параболы $k=\left(h-\frac{l^{2}}{2}\right)^{2}$, выделяемая условием $\frac{l^{2}}{2} \leqslant$ $h \leqslant \frac{l^{2}}{2}+\frac{1}{l^{2}} ;$ участки $\beta_{1}, \beta_{2}, \beta_{3}$ на рис. 11 ; 


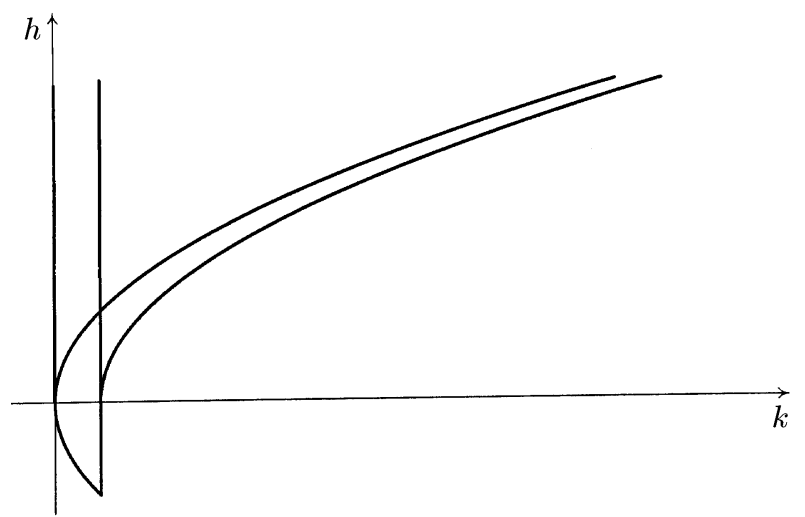

Рис. 9a). $l=0$

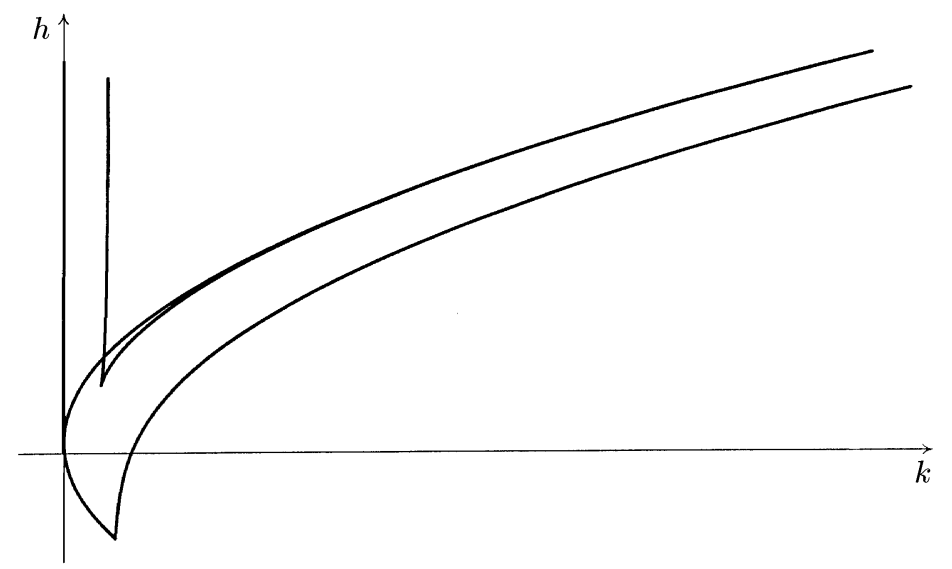

Рис. 9б). $0<l<\sqrt{2}$

Класс IV: кривая, задаваемая параметрически:

$$
k=1+l p+p^{4}, \quad h=p^{2}-\frac{l}{2} p ;
$$

часть с положительным $p \geqslant \frac{l}{2}$ - это участок $\gamma_{1}$ на рис. 11 , различные куски с $p \in(-\infty, 0)-$ это $\gamma_{2}, \ldots, \gamma_{7}$.

Точка Н внизу каждой бифуркационной диаграммы имеет координаты $(h, k)=$ $\left(\frac{l^{2}}{4}-1,\left(\frac{l^{2}}{4}+1\right)^{2}\right)$. Это спящий волчок в подвешенном состоянии. Левая граница образа отображения момента состоит из линий классов I и II, правая граница $k_{\text {max задается кривой }} \gamma_{1}$ из класса IV. Kлассы III и IV (исключая $\gamma_{1}$ ) определяют внутренние линии.

Используя стандартные обозначения для невырожденных бифуркаций [11], [13], [23], мы указываем на рис. 10, какой тип перестройки торов реализуется на каждом участке бифуркационной диаграммы. Система Ковалевской содержит бифуркации четырех типов

$$
A, A^{*}, B, C_{2},
$$




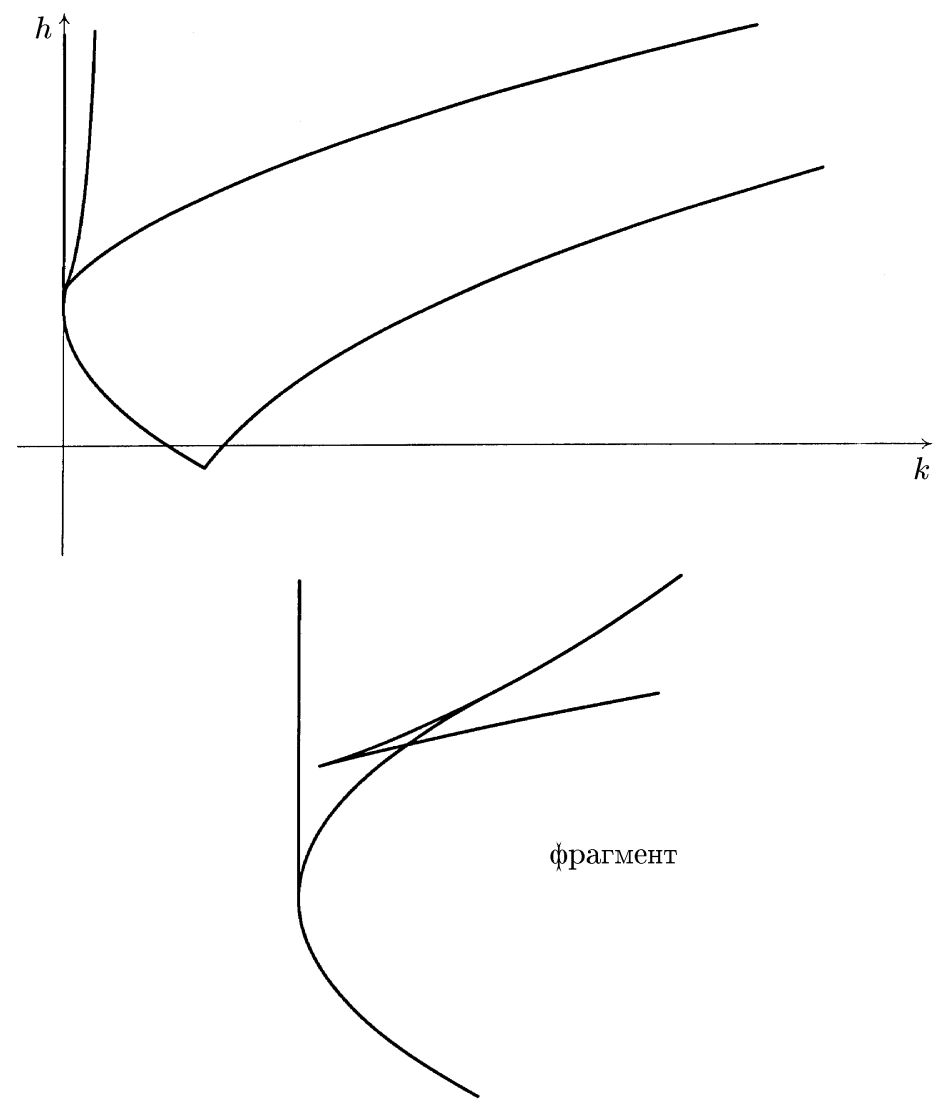

Рис. 9в). $\sqrt{2}<l<\frac{4}{3} \sqrt[4]{3}$

топология которых показана на рис. 12. Следующий список содержит информацию, уже накопленную в литературе:

$\begin{array}{cc}\text { участок } & \text { тип бифуркации } \\ \delta_{1} & A+A \\ \delta_{2} & A+A \\ \alpha_{1} & A \\ \alpha_{2} & A+A \\ \beta_{1} & B \\ \beta_{2} & C_{2} \\ \beta_{3} & B+B\end{array}$

$\begin{array}{cc}\text { участок } & \text { тип бифуркации } \\ \gamma_{1} & A \\ \gamma_{2} & A^{*}+A^{*} \\ \gamma_{3} & B \\ \gamma_{4} & A \\ \gamma_{5} & B \\ \gamma_{6} & B+B \\ \gamma_{7} & A+A\end{array}$

Используя эту информацию, можно непосредственно построить молекулы $W^{*}(\tau)$ для допустимых кривых $\tau$ в $\mathbb{R}^{2}(h, k)$. В частности, молекулы для фиксированных значений энергии $(h, l)$ и меняющегося $k$, отвечающие областям на рис. 8, могут быть построены для каждого из десяти типов слоения: достаточно просто двигаться по горизонтальным линиям с метками А, B, ..., J на рис. 10 и учитывать типы перестроек, происходящих при пересечении участков бифуркационной диаграммы. Табл. 3 содержит полный список. 


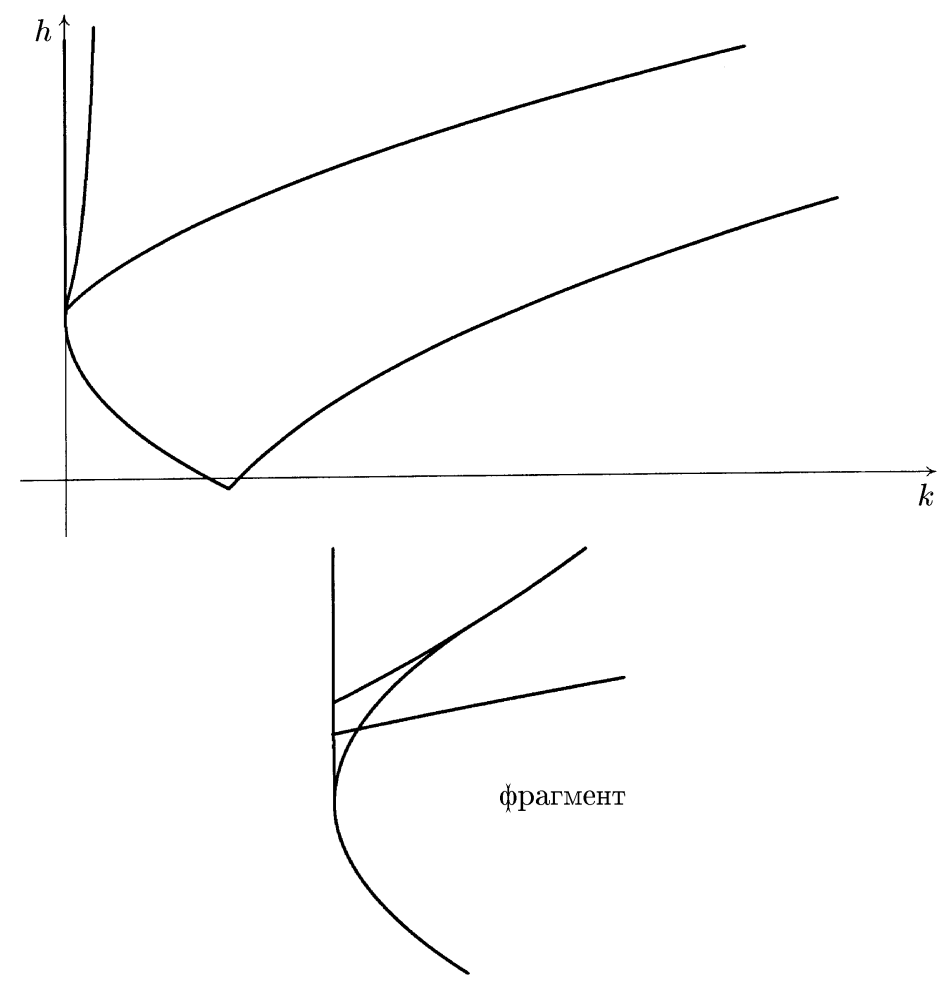

Рис. 9г). $\frac{4}{3} \sqrt[4]{3}<l<2$

Кривые критических значений разбивают образ отображения момента на несколько областей регулярности, внутри которых имеющиеся торы не испытывают никаких бифуркаций. В полной бифуркационной диаграмме $\Sigma_{h, k, l}$ кривые из $\Sigma_{h, k}^{l}$, двигаясь в $l$-направлении, заметают двумерные поверхности; эти поверхности определяют пять различных областей (i), (ii), (iii), (iv), (v) в $\mathbb{R}^{3}(h, k, l)$, названных в [16] областями Харламова. Отметим, однако, что прообраз данной точки $(h, k, l)$ может состоять из нескольких торов Лиувилля, некоторые из которых могут быть связаны симметриями, а некоторые - нет. Поэтому с топологической точки зрения мы предпочитаем рассматривать сами семейства торов Лиувилля вместо их образов при отображении момента. Оказывается, с точностью до симметрии имеется также пять различных семейств лиувиллевых торов, которые мы обозначим через $(1),(2),(3),(4),(5)$. В одну и ту же область Харламова могут отображаться несколько различных семейств. Точная схема такова:

$\begin{array}{ccr}\text { семейства торов } & & \text { области Ха } \\ (1) & \longrightarrow & \text { (i) } \\ \text { два (2) } & \longrightarrow & \text { (ii) } \\ \text { два (3) } & \longrightarrow & \text { (iii) } \\ (1) \text { и (4) } & \longrightarrow & \text { (iv) } \\ \text { два (2) и два (5) } & \longrightarrow & \text { (v) }\end{array}$

Сечения областей Харламова при данном значении $l$ видны на диаграмме $\Sigma_{h, k}^{l}$, а 


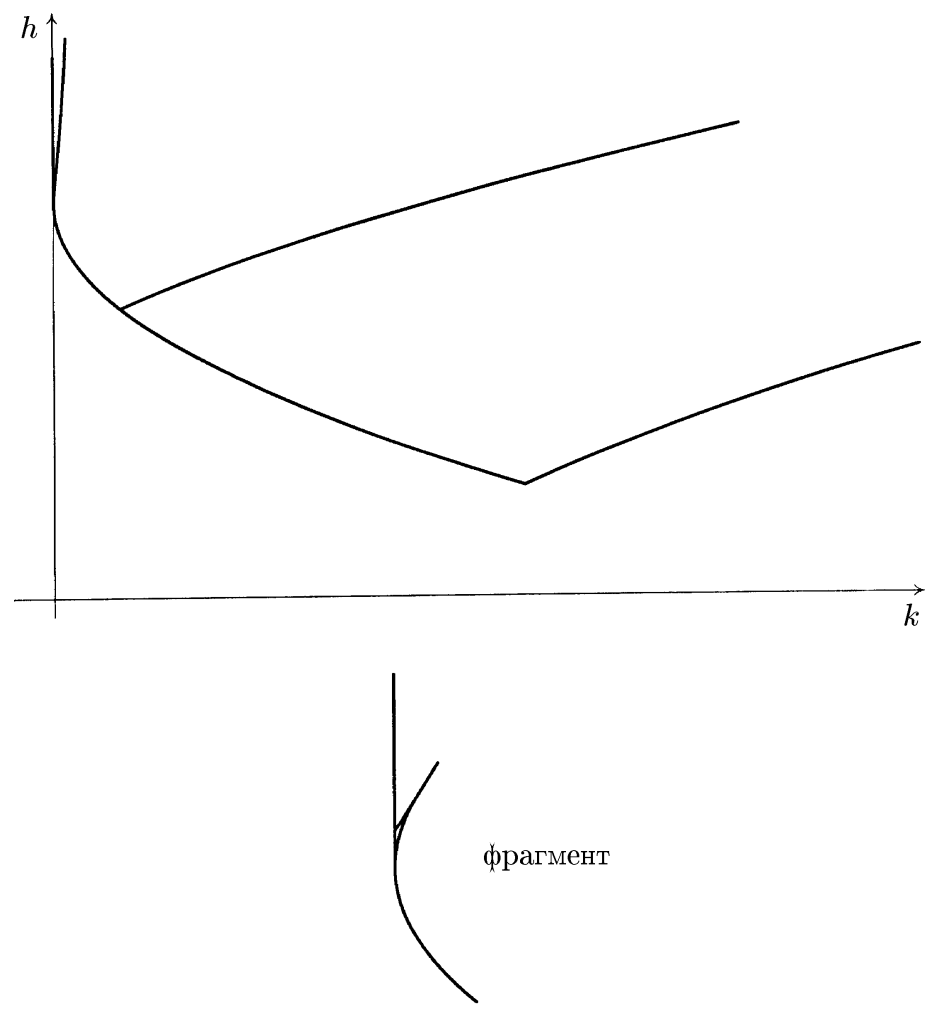

Рис. 9д). $l>2$

на рис. 11 указано, какие именно семейства торов отображаются в них.

Чтобы понять взаимосвязь между бифуркационньми диаграммами $\Sigma_{h, k}^{l}$ и бифуркациями в семействах торов Лиувилля, рассмотрим участки $\gamma_{3}, \gamma_{4}$ на рис. 11б). Когда точка $(h, k)$ пересекает $\gamma_{4}$, двигаясь справа налево, с семейством (1) ничего не происходит. Бифуркация $A$, указанная на рис. 10б), влияет только на семейство (4), которое рождается при этом переходе. При дальнейшем уменьшении $k$ торы из семейств (1) и (4) принимают участие в бифуркации типа $B$ на участке $\gamma_{3}$.

С другой стороны, отметим, что на рис. 11 некоторым участкам соответствуют два обозначения: $\gamma_{1}, \gamma_{4}$ на рис. $\left.11 \mathrm{a}\right)$ и $\delta_{1}, \delta_{2}$ на рис. $\left.11 \Gamma\right)$, д). Причина в том, что два различных семейства торов независимо друг от друга подвергаются перестройке при переходе через один и тот же участок бифуркационной диаграммы $\mathbb{R}^{2}(h, k)$.

Каждая бифуркационная диаграмма $\Sigma_{h, k}^{l}$ имеет множество особых точек $\Sigma_{0}^{l}$. На рис. 10 они видны как точки пересечения, касания или возврата. Это особое множество находится в естественном соответствии с кривыми бифуркационной диаграммы $\Sigma_{h, l}$ на рис. 8 , которые мы можем получить из особых точек, показанных на рис. 10 и 11, варьируя параметр $l$ и указывая соответствующие значения энергии $h$ на плоскости $\mathbb{R}^{2}(h, l)$ как функции от $l$. Жирные точки $\mathrm{H}, \mathrm{U}_{1}, \mathrm{U}_{2}, \mathrm{U}_{3}$, $\mathrm{M}_{1}, \mathrm{M}_{2}$ на рис. 10, 11 отвечают положениям равновесия и дают жирные сплошные линии Н, U, М на рис. 8 соответственно. Их топологическое строение задается 


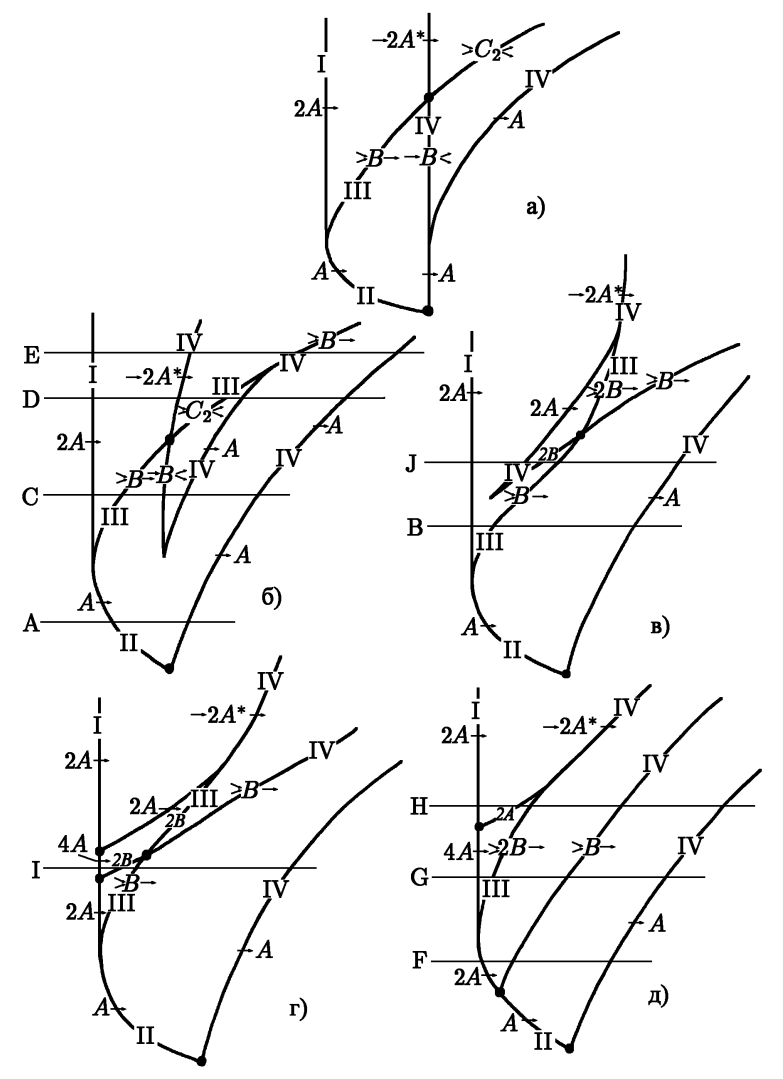

Рис. 10

следуюшим списком [26], [27]:

$\begin{array}{ccc}\text { точка } & \text { тип } & \text { топологическая структура } \\ \mathrm{H} & \text { центр-центр } & A \times A \\ \mathrm{U}_{1} & \text { седло-седло } & \left(B \times C_{2}\right) / \mathbb{Z}_{2} \\ \mathrm{U}_{2} & \text { седло-седло } & B \times B \\ \mathrm{U}_{3} & \text { центр-седло } & A \times B \\ \mathrm{M}_{1} & \text { центр-центр } & 2(A \times A) \\ \mathrm{M}_{2} & \text { центр-седло } & A \times B\end{array}$

Особенностей типа фокус-фокус в случае Ковалевской нет.

Остальные особые точки соответствуют вырожденным замкнутым траекториям различных типов; при изменении $l$ они образуют кривые e, c, h на диаграмме $\Sigma_{h, k, l}$ : кривая е происходит из точки, в которой встречаются классы I, II и III; кривая с возникает из точки возврата при $p=-\left(\frac{l}{4}\right)^{1 / 3}$ на кривой IV; кривая $\mathrm{h}$ получается из точек, где класс III заканчивается и касается класса IV $(p=-1 / l)$. Но в зависимости от значения $l$ окрестность этих точек может быть различна. Поэтому для каждого случая выделяются два типа. Следуюший список содержит 


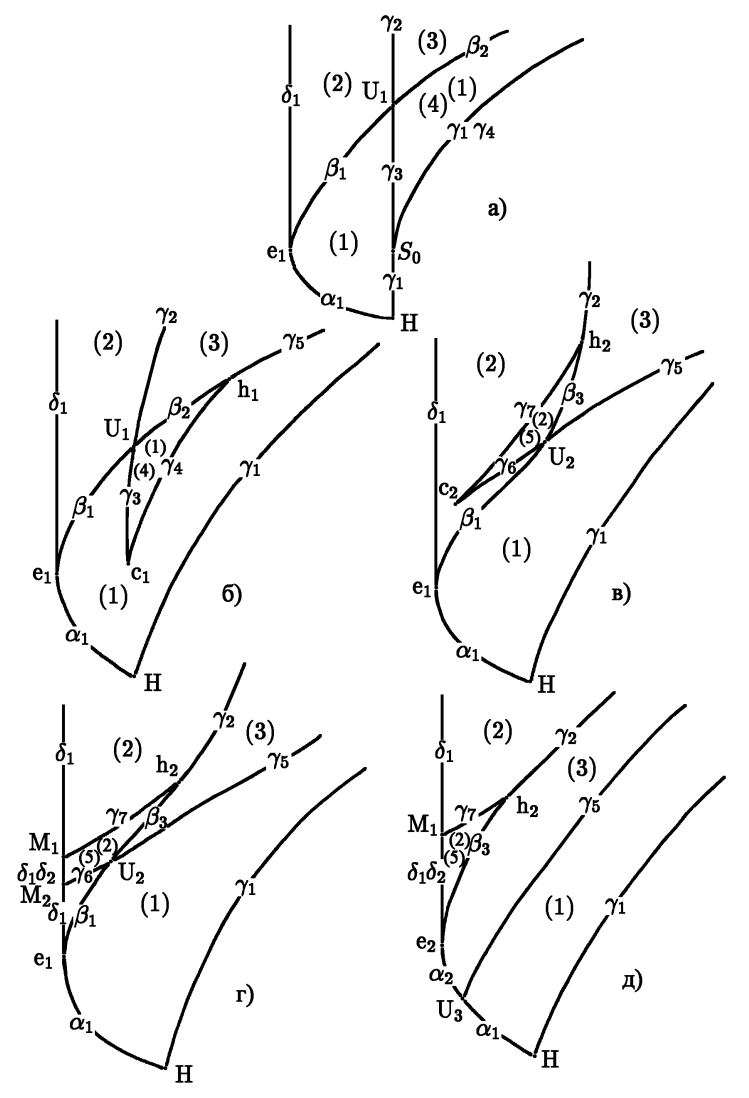

Рис. 11

известную информацию об их характере:

$$
\begin{array}{cc}
\text { особая точка } & \text { область изменения } l \\
S_{0} & l=0 \\
\mathrm{e}_{1} & 0<|l|<2 \\
\mathrm{e}_{2} & |l|>2 \\
\mathrm{c}_{1} & 0<|l|<\sqrt{2} \\
\mathrm{c}_{2} & \sqrt{2}<|l|<\frac{4}{3} \sqrt[4]{3} \\
\mathrm{~h}_{1} & 0<|l|<\sqrt{2} \\
\mathrm{~h}_{2} & |l|>\sqrt{2}
\end{array}
$$

тип вырождения

эллиптическая вилка эллиптическая вилка эллиптическая вилка седло-узел седло-узел гиперболическая вилка гиперболическое удвоение периода

Эллиптического удвоения периода в случае Ковалевской не возникает.

Используя информацию о топологическом характере бифуркаций, отвечающих классам Аппельрота и их вырождениям, мы можем построить круговые молекулы $W^{*}\left(y_{0}\right)$ для каждой из 13 особых точек. Это сделано в табл. 4. Поясним обозначения на следуюшем примере:

$$
\stackrel{(2)}{\stackrel{(5)}{\longrightarrow}} B \gamma_{6} \stackrel{(2)}{\longrightarrow} \text {. }
$$




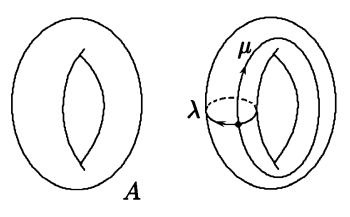

a)

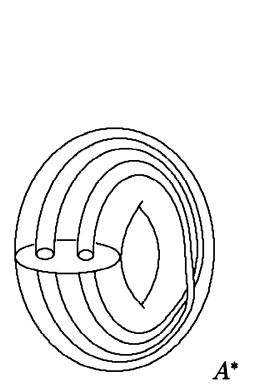

$A^{*}$
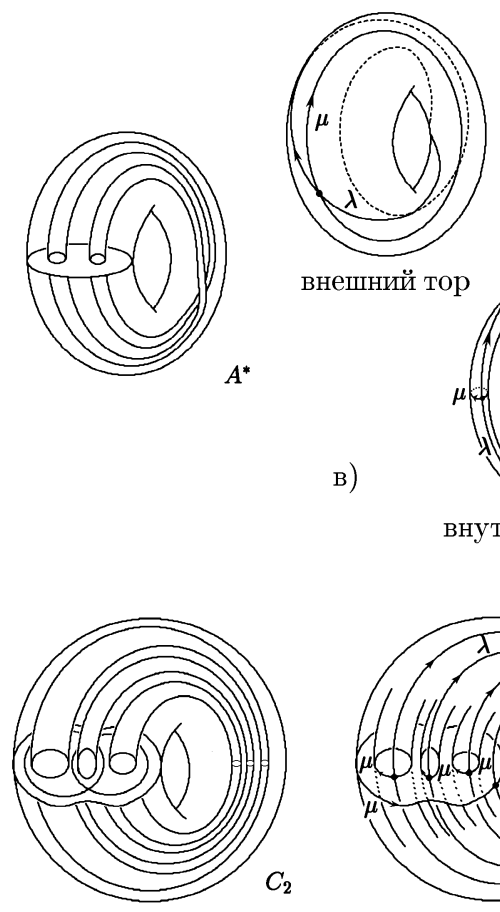

внешний тор

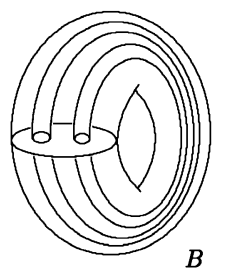

б)

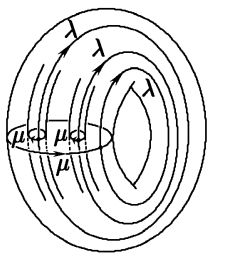

в)
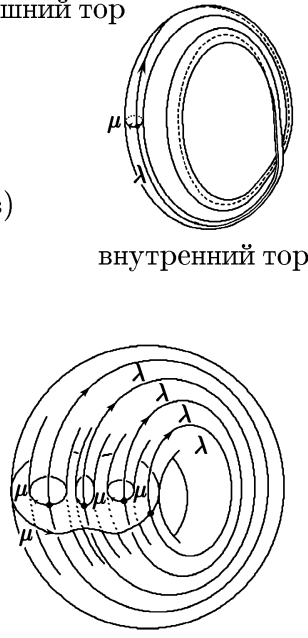

г)

Рис. 12

Главное здесь - это атом $B$. Направление стрелок указывает на возрастание $k$. Номера над стрелками обозначают семейства торов, соответствующие данному ребру. Индекс $\gamma_{6}$ говорит, на каком участке $\Sigma_{h, k}$ появляется (т.е. какому классу Аппельрота соответствует) данная бифуркация $B$.

\section{§4. Топологическая структура слоения Лиувилля в случае Ковалевской}

Как мы уже отмечали вьше, в случае Ковалевской имеется 10 различных типов слоений Лиувилля на изоэнергетических поверхностях. Эти типы мы обозначили через А, В, .. , Ј. Грубые инварианты этих слоений (так называемые молекулы) были исследованы в целой серии работ [28], [12], [15], [16], [29]. Некоторая информация о числовых метках этих молекул была получена в работах [27], [14]. Следующая теорема подводит итог этому циклу исследований и дает полное опи- 
ТАБлицА 4. Круговые молекулы случая Ковалевской

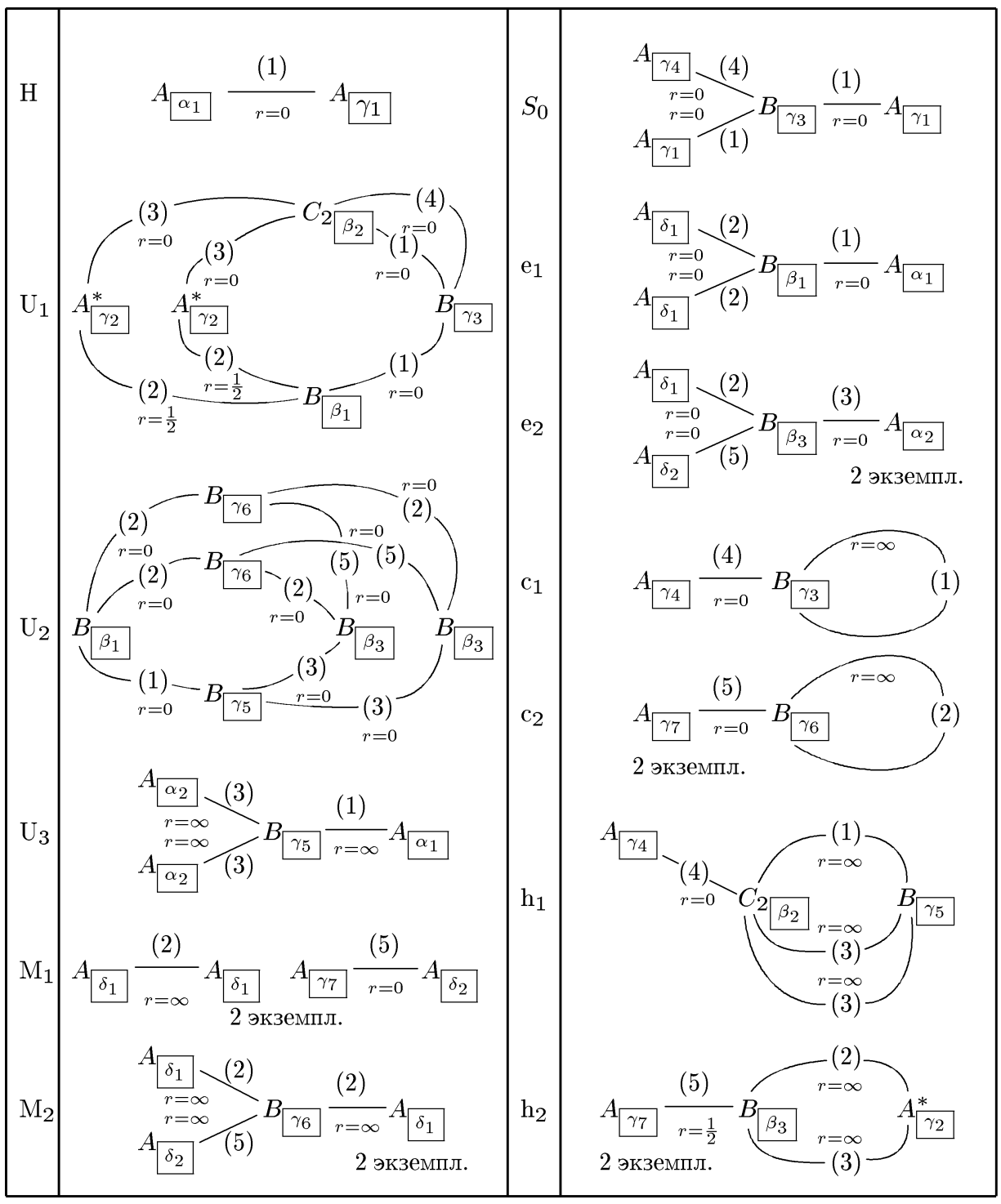

сание топологии всех возможных слоений Лиувилля в терминах так называемых меченых молекул, являющихся их полными топологическими инвариантами (см. $[13],[23])$.

ТЕОРЕМА. Полный список всех меченых молекул, возникающих в случае Ковалевской, состоит из десяти случаев, перечисленных в табл. 3.

КомментАРий. Мы также указываем ниже матрицы склейки, отвечающие каждому ребру молекулы. В действительности указание матриц даже более интересно, чем числовые метки. В определенном смысле матрицы склейки являются "основной информацией" о слоении Лиувилля. 
Поскольку молекулы без меток для случая Ковалевской уже известны, нам остается найти числовые метки $r, \varepsilon, n$. Наиболее естественный способ это сделать - использовать информацию о круговых молекулах особых точек бифуркационной диаграммы отображения момента.

Разобьем доказательство теоремы на несколько шагов (п.п. 4.1-4.4).

4.1. Допустимые системы координат на торах Лиувилля. Каждьй из гладких участков $\gamma_{1}, \gamma_{2}, \ldots$ бифуркационной диаграммы соответствует некоторой бифуркации торов Лиувилля (см. вьше). Рассмотрим переход через такую бифуркацию, взяв прообраз гладкого отрезка, трансверсально пересекающего бифуркационную диаграмму $\Sigma$. С топологической точки зрения этот прообраз является трехмерным многообразием $Q_{i}$, граница которого состоит из торов Лиувилля. Обычно мы называем такое многообразие 3-атомом. На каждом из граничных торов $Q_{i}$ мы можем задать пару базисных циклов $(\lambda, \mu)$, которые образуют (если их рассматривать совместно) так называемую допустимую систему координат на $\partial Q_{i}$. Формальное определение допустимой системы координат можно найти в работах [23], [11].

Для атомов (бифуркаций), возникающих в случае Ковалевской, они показаны на рис. 12.

Для атома $A$ многообразие $Q$ является полноторием с эллиптической орбитой в качестве оси. Для атома $B$ мы имеем полноторие, окружающее гиперболическую орбиту, с двумя вырезанными менњшими полноториями. Эти торические дырки не зацеплены с гиперболической орбитой и между собой. В случае атома $A^{*}$ многообразие $Q$ снова является полноторием, идущим вдоль гиперболической орбиты, из которого вырезано тонкое полноторие, два раза проходящее вдоль оси (т.е. гиперболической орбиты) и зацепленное с ней с индексом 2. Наконец, атом $C_{2}$ соответствует полноторию, из которого вырезаны три тонких взаимно незацепленных полнотория, каждое из которых обходит вокруг оси один раз.

Отметим, что первый базисный цикл $\lambda$ всегда определен однозначно. В случае бифуркаций $A, B, C_{2}$ гиперболического типа цикл $\lambda$ может рассматриваться как неустойчивая периодическая орбита, сдвинутая на тор Лиувилля (в случае атома $C_{2}$ можно рассмотреть любую из двух таких орбит, поскольку они гомотопны). Если бифуркация имеет тип $A$, то $\lambda$ - это цикл, сжимающийся в точку при подходе к эллиптической орбите. Цикл $\mu$ в отличие от $\lambda$ может определяться многими способами.

Через $\lambda_{i}$ ниже мы будем обозначать первый базисный цикл, соответствующий атому $Q_{i}$. При этом в каждом конкретном случае для системы Ковалевской индекс $i$ будет заменяться на один из 14 символов $\delta_{1}, \ldots, \gamma_{7}$.

Итак, для продолжения анализа мы должны выбрать некоторую допустимую систему координат для каждой бифуркации $\delta_{1}, \ldots, \gamma_{7}$. Это даст пару циклов $\left(\lambda_{i}, \mu_{i}\right)$ на каждом семействе торов Лиувилля. Напомним, что циклы $\lambda_{i}$ не меняются при бифуркациях гиперболического типа (они исчезают вместе с семейством при эллиптической бифуркации $A$ ). Что касается циклов $\mu$, то они выбираются таким образом, что все они, грубо говоря, лежат на подходящем сечении рассматриваемого атома.

Если данное семейство торов Лиувилля подвергается различньм бифуркациям $\gamma_{i}$ и $\gamma_{j}$, то эта процедура порождает, вообще говоря, различные пары базис- 
ных циклов $\left(\lambda_{i}, \mu_{i}\right)$ и $\left(\lambda_{j}, \mu_{j}\right)$ из фундаментальной группы торов семейства. Таким образом, на одном и том же торе семейства возникает много различных циклов. Оказывается, однако, что цикл $\mu_{i}$ может быть выбран совпадаюшим с некоторьм $\lambda_{j}$, определенным другой бифуркацией (или может быть выражен через такие циклы естественным образом). Это обстоятельство является весьма существеным: в наших конструкциях мы можем обойтись лишш однозначно определенными циклами $\lambda_{i}$. Поясним это на следуюшем примере.

Рассмотрим особую точку $\mathrm{U}_{2} \in \Sigma$. Соответствующая особенность имеет тип седло-седло и может быть представлена как прямое произведение двух простейших седловых бифуркаций $B \times B$ (рис. 13). Обозначим граничные циклы двух утолшенных восьмерок $P$ и $P^{\prime}$ через $\alpha, \beta, \gamma$ и $\alpha^{\prime}, \beta^{\prime}, \gamma^{\prime}$, как показано на рис. 13.

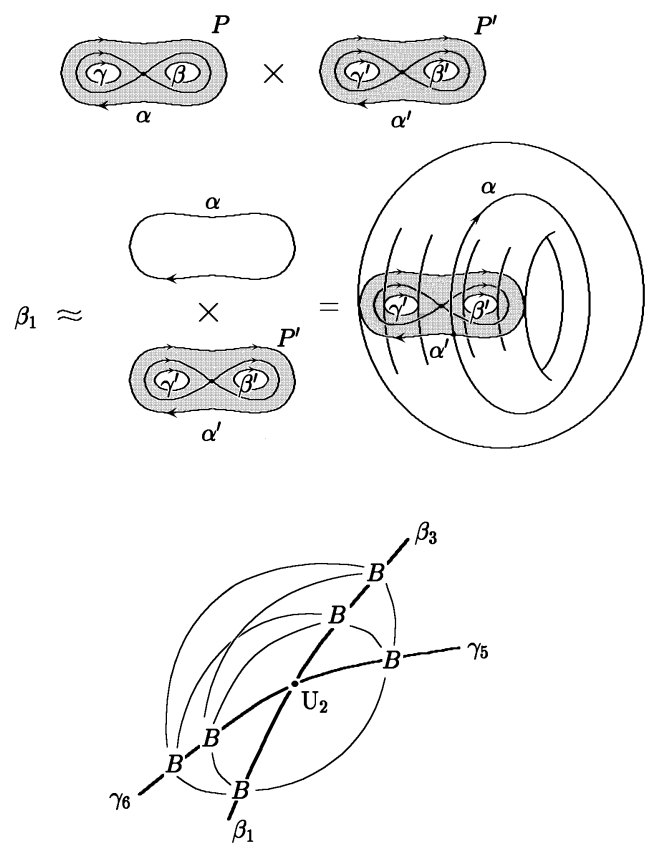

Рис. 13

Бифуркации $\beta_{1}, \gamma_{5}, \beta_{3}, \gamma_{6}$, расположенные вокруг этой точки, могут быть описаны следующим образом с использованием структуры прямого произведения $P \times P^{\prime}:$

$Q_{\beta_{1}}$ получается взятием прямого произведения внешней граничной окружности $\alpha$ поверхности $P$ на всю утолшенную восьмерку $P^{\prime}$,

$Q_{\beta_{3}}$ получается взятием прямого произведения двух внутренних граничных окружностей $\beta$ и $\gamma$ поверхности $P$ на всю утолщенную восьмерку $P^{\prime}$,

$Q_{\gamma_{5}}$ получается взятием прямого произведения внешней граничной окружности $\alpha^{\prime}$ поверхности $P^{\prime}$ на всю утолшенную восьмерку $P$,

$Q_{\gamma_{6}}$ получается взятием прямого произведения двух внутренних граничных окружностей $\beta^{\prime}$ и $\gamma^{\prime}$ поверхности $P$ на всю утолшенную восьмерку $P$. 
Это утверждение является простой переформулировкой того факта, что особенность $\mathrm{U}_{2}$ имеет тип прямого произведения $B \times B$.

После этого описания ясно, какие циклы следует взять в качестве допустимых систем координат. Например, для бифуркации $\beta_{1}$ мы имеем три граничных тора (рис. 13). В качестве базисных циклов на них мы можем взять $\left(\alpha,-\alpha^{\prime}\right),\left(\alpha, \beta^{\prime}\right)$ и $\left(\alpha, \gamma^{\prime}\right)$ соответственно. (Знак минус требуется для того, чтобы согласовать ориентации на торах.)

Для оставшихся трех случаев картина совершенно аналогична. Остается заметить, что цикл $\alpha$ в нашем случае в точности совпадает с циклом $\lambda_{\beta_{1}}$ (т.е. с первьм базисным циклом для $\beta_{1}$ ). То же самое верно для остальных бифуркаций: $\alpha^{\prime}=\lambda_{\gamma_{5}}, \beta=\lambda_{\beta_{3}}, \gamma=\lambda_{\beta_{3}}, \beta^{\prime}=\lambda_{\gamma_{6}}, \gamma^{\prime}=\lambda_{\gamma_{6}}$.

В результате мы имеем следующий список допустимых систем координат для бифуркаций $\gamma_{6}, \beta_{1}, \gamma_{5}, \beta_{3}$ :

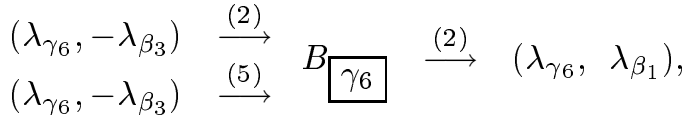

$$
\begin{aligned}
& \begin{array}{lll}
\left(\lambda_{\beta_{1}},\right. & \left.\lambda_{\gamma_{6}}\right) \stackrel{(2)}{\longrightarrow} \\
\left(\lambda_{\beta_{1}},\right. & \left.\lambda_{\gamma_{6}}\right) \stackrel{(2)}{\longrightarrow}
\end{array} \stackrel{\beta_{1}}{\longrightarrow} \stackrel{(1)}{\longrightarrow}\left(\lambda_{\beta_{1}},-\lambda_{\gamma_{5}}\right), \\
& \begin{array}{ll}
\left(\lambda_{\gamma_{5}},-\lambda_{\beta_{3}}\right) & \stackrel{(3)}{\longrightarrow} \\
\left(\lambda_{\gamma_{5}},-\lambda_{\beta_{3}}\right) & \stackrel{(3)}{\longrightarrow}
\end{array} \\
& \begin{array}{lll}
\left(\lambda_{\beta_{3}},\right. & \left.\lambda_{\gamma_{6}}\right) & \stackrel{(2)}{\longrightarrow} \\
\left(\lambda_{\beta_{3}},\right. & \left.\lambda_{\gamma_{6}}\right) \stackrel{(5)}{\longrightarrow}
\end{array} \stackrel{\beta_{3}}{\longrightarrow} \stackrel{(3)}{\longrightarrow}\left(\lambda_{\beta_{3}},-\lambda_{\gamma_{5}}\right) .
\end{aligned}
$$

Здесь стрелки на ребрах указывают направление возрастания интеграла $K$. Цифры над ребрами показывают номера соответствующих семейств торов Лиувилля.

Подобный анализ несложно провести для остальных случаев. В качестве следуюшего примера мы сформулируем результат для особой точки $\mathrm{U}_{1}$, которая устроена немного сложнее, поскольку она имеет тип не "прямого", а "почти прямого" произведения $\left(B \times C_{2}\right) / \mathbb{Z}_{2}$ (см. [21]). Допустимые системы координат таковы:

$$
\begin{aligned}
& \left(\lambda_{\gamma_{2}}, \frac{\lambda_{\beta_{1}}+\lambda_{\gamma_{2}}}{2}\right) \stackrel{(2)}{\longrightarrow} A_{\gamma_{2}}^{*} \stackrel{(3)}{\longrightarrow}\left(\lambda_{\gamma_{2}},-\lambda_{\beta_{2}}\right),
\end{aligned}
$$

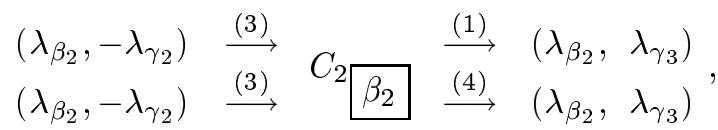

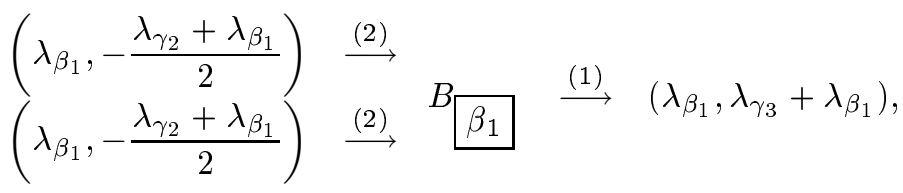

$$
\begin{aligned}
& \left(\begin{array}{lll}
\lambda_{\gamma_{3}}, & \left.\lambda_{\beta_{1}}\right) \stackrel{(1)}{\longrightarrow} B \stackrel{(1)}{\longrightarrow}\left(\lambda_{\gamma_{3}}, \stackrel{(4)}{\longrightarrow}\left(\lambda_{\gamma_{3}},-\lambda_{\beta_{2}}\right)\right.
\end{array}\right)
\end{aligned}
$$


Это полная информация о допустимых системах координат на седловых атомах.

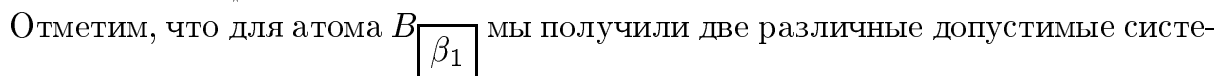
мы координат. Это не должно нас удивлять, поскольку, как уже было объяснено, допустимые системы координат определены неоднозначно.

Здесь мы должны сделать определенный выбор. Наша конечная цель - найти множество матрицсклеек (или меток) для круговых и изоэнергетических молекул. При этом мы будем использовать одну и ту же допустимую систему для данной бифуркации $i$, где бы она не появлялась. В частности, для бифуркации $B \sqrt{\beta_{1}}$

мы будем использовать первую допустимую систему координат, возникшую из точки $\mathrm{U}_{2}$. При этом мы должны будем объяснить, как связаны между собой две возникшие системы координат, т.е. как цикл $\left(\lambda_{\beta_{1}}+\lambda_{\gamma_{2}}\right) / 2$ выражается через $\lambda_{\beta_{1}}$ и $\lambda_{\gamma_{6}}, \lambda_{\gamma_{3}}$ через $\lambda_{\beta_{1}}$ и $\lambda_{\gamma_{5}}$. Ответ на этот вопрос будет дан в следующем пункте.

В принципе наш глобальный анализ может начинаться с любой особой точки (например, с Н), а затем продолжаться шаг за шагом вдоль линий бифуркационной диаграммы от одной особой точки до другой. Матрицы склейки, которые мы в результате получим, - но не метки $r, \varepsilon, n$ - будут зависеть от выбора начальной точки. В рассматриваемом случае наилучшая стратегия состоит в том, чтобы начать с особой точки, расположенной где-то в центре бифуркационной диаграммы. В качестве такой точки мы выбрали точку $\mathrm{U}_{2}$.

Начав с окрестностей точек $\mathrm{U}_{2}$ и $\mathrm{U}_{1}$, мы определили пока допустимые системы координат на атомах, относящиеся к семи гиперболическим бифуркациям $\beta_{1}, \beta_{2}$, $\beta_{3}, \gamma_{2}, \gamma_{3}, \gamma_{5}, \gamma_{6}$. Оставшиеся бифуркации имеют эллиптический тип $A$.

Мы можем проанализировать $Q_{\gamma_{1}}$ и $Q_{\gamma_{4}}$, рассматривая круговую молекулу точки $S_{0} ; Q_{\alpha_{1}}$ и $Q_{\delta_{1}}-$ с помощью особой точки е 1 ; $Q_{\alpha_{2}}$ и $Q_{\delta_{2}}-$ с помощью е 2 .

Все перечисленные точки имеют тип эллиптической вилки. Поэтому (как это видно из рис. 5) в качестве второго базисного цикла мы можем взять ось соседнего атома $B$.

Наконец, для атома $Q_{\gamma_{7}}$ допустимую систему координат можно получить, используя точку $\mathrm{h}_{2}$, которая имеет тип удвоения периода, второй базисный цикл $\mu$ может быть задан формулой $\left(\lambda+\lambda^{\prime}\right) / 2$, где $\lambda^{\prime}-$ это ось соседнего атома $B$ (см. рис. 6).

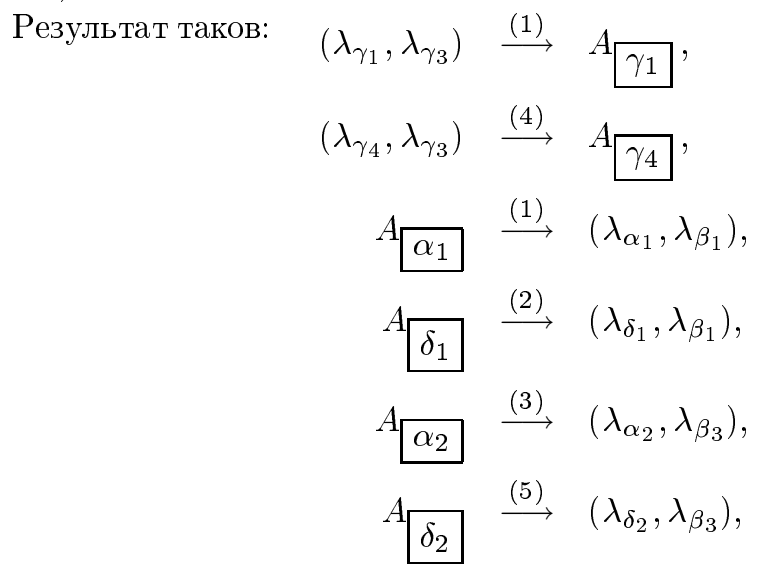




$$
A_{\gamma_{7}} \stackrel{(5)}{\longrightarrow}\left(\lambda_{\gamma_{7}}, \frac{\lambda_{\beta_{3}}+\lambda_{\gamma_{7}}}{2}\right) .
$$

4.2. Взаимное расположение базисных циклов на торах Лиувилля. Задача, которую мы в конце концов хотим решить, состоит в описании матриц склейки, которые представляют собой матрицы перехода между допустимыми системами координат. Поясним эту задачу на простом примере.

Рассмотрим изоэнергетическую поверхность, соответствуюшую малым значениям энергии. В наших обозначениях соответствуюшая молекула имеет вид

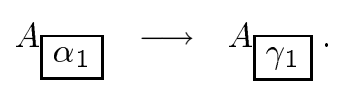

Для каждого из этих двух атомов мы уже знаем допустимые системы координат. Это соответственно $\left(\lambda_{\alpha_{1}}, \lambda_{\beta_{1}}\right)$ и $\left(\lambda_{\gamma_{1}}, \lambda_{\gamma_{3}}\right)$. Нам требуется найти матрицу склейки между этими атомами, т.е. согласно определению матрицу перехода $C=\left(c_{i j}\right)$, определяемую соотношением

$$
\left(\begin{array}{l}
\lambda_{\gamma_{1}} \\
\lambda_{\gamma_{3}}
\end{array}\right)=\left(\begin{array}{ll}
c_{11} & c_{12} \\
c_{21} & c_{22}
\end{array}\right)\left(\begin{array}{c}
\lambda_{\alpha_{1}} \\
\lambda_{\beta_{1}}
\end{array}\right)
$$

Чтобы найти эту матрицу, мы должны считать, что все базисные циклы расположены на одном и том же торе Лиувилля. Это действительно так. В самом деле, рассмотрим произвольный тор Лиувилля $T$ из семейства (1). Из рис. 10, 11 видно, что этот тор может быть подвергнут бифуркациям $A \gamma_{1}, A \alpha_{1}, B \beta_{1}, B \gamma_{3}$, $B_{\gamma_{5}}, C_{2} \beta_{2}$. Когда $T$ расположен вблизи некоторой бифуркации, эта бифуркация позволяет определить цикл $\lambda$ на $T$, который всегда однозначно определен. Если эта бифуркация имеет гиперболический тип, то $\lambda$ является неустойчивой (гиперболической) периодической орбитой, с двинутой на этот тор. Если же бифуркация имеет эллиптический тип (т.е. соответствует атому $A$ ), то $\lambda$ - это цикл, сжимающийся в точку. Для семейства (1) это в точности циклы, обозначенные через $\lambda_{\gamma_{1}}, \lambda_{\alpha_{1}}, \lambda_{\beta_{1}}, \lambda_{\beta_{2}}, \lambda_{\gamma_{3}}, \lambda_{\gamma_{5}}$. Таким образом, мы можем считать, что все эти циклы лежат на одном и том же торе Лиувилля.

Вопрос теперь состоит в том, как они взаимно расположены. Если мы ответим на этот вопрос, то мы автоматически сможем найти все матрицы склейки.

Таким образом, для каждого из пяти семейств торов Лиувилля нам требуется описать взаимное расположение циклов $\lambda$. Разумеется, мы имеем в виду только те циклы, которые имеют отношение к рассматриваемому семейству. Например, нет никакого смысла в том, чтобы обсуждать расположение цикла $\lambda_{\gamma_{1}}$ на торах из семейства (3), поскольку этот цикл расположен "вдалеке от этого семейства".

Оказывается, эта задача может быть решена, если мы соберем вместе всю информацию о циклах, которая нам известна из круговых молекул и допустимых систем координат. Наиболее важная информация, которая извлекается из круговых молекул, относится к индексам пересечения соответствующих циклов.

В качестве примера рассмотрим точку е ${ }_{1}$. Она имеет тип вилки. Соответствующая круговая молекулапоказана в табл. 4 . Все $r$-метки на ее ребрах равны нулю. 
Это в точности означает, что циклы $\lambda_{\alpha_{1}}, \lambda_{\beta_{1}}$ имеют индекс пересечения 1 в семействе (1), а циклы $\lambda_{\alpha_{1}}, \lambda_{\delta_{1}}$ имеют индекс пересечения 1 в семействе (2).

Тот же самый результат может быть получен из рис. 5 , показывающего бифуркацию типа вилка. Однако мы предпочитаем использовать в наших рассуждениях круговые молекулы, поскольку все они взяты из стандартного списка, что делает процедуру вычисления более простой и формальной.

Напомним простое правило, устанавливающее связь между $r$-метками на ребрах молекулы и индексами пересечения соответствующих циклов [23], [27]. Рассмотрим ребро молекулы и два цикла $\lambda^{+}$и $\lambda^{-}$, которые являются однозначно определенными базисньми циклами на атомах, соединенных данньм ребром. Тогда правило формулируется следующим образом:

1) если $r=0$, то $\lambda^{+}$и $\lambda^{-}$имеют индекс пересечения 1 ;

2) если $r=\infty$, то $\lambda^{+}$и $\lambda^{-}$имеют индекс пересечения 0 , т.е. гомологичны (с точностью до знака);

3) если $r=\frac{1}{2}$, то $\lambda^{+}$и $\lambda^{-}$имеют индекс пересечения 2.

Проанализируем каждое семейство по отдельности.

Семейство (1).

Бифуркации, относящиеся к этому семейству, -это $A\left[\gamma_{1}, A \alpha_{1}, B \beta_{1}, B\left[\gamma_{3}\right.\right.$,

\section{$B_{\gamma_{5}}, C_{2} \beta_{2}$}

Особые точки, относящиеся к этому семейству, - это $\mathrm{H}, \mathrm{e}_{1}, \mathrm{U}_{1}, \mathrm{~S}_{0}, \mathrm{~h}_{1}, \mathrm{U}_{2}, \mathrm{U}_{3}$.

Информация, которую мы можем извлечь из круговых молекул, такова.

Точка Н: $\lambda_{\gamma_{1}}$ и $\lambda_{\alpha_{1}}$ имеют индекс пересечения 1.

Точка $e_{1}: \lambda_{\alpha_{1}}$ и $\lambda_{\beta_{1}}$ имеют индекс пересечения 1 .

Точка $\mathrm{U}_{1}: \lambda_{\beta_{2}}$ и $\lambda_{\gamma_{3}}$ имеют индекс пересечения 1 , так же как и циклы $\lambda_{\gamma_{3}}$ и $\lambda_{\beta_{1}}$.

Точка $\mathrm{S}_{0}: \lambda_{\gamma_{3}}$ и $\lambda_{\gamma_{1}}$ имеют индекс пересечения 1.

Точка $\mathrm{h}_{1}: \lambda_{\beta_{2}}$ и $\lambda_{\gamma_{5}}$ имеют индекс пересечения 0 , т.е. гомологичны.

Точка $\mathrm{U}_{2}: \lambda_{\beta_{1}}$ и $\lambda_{\gamma_{5}}$ имеют индекс пересечения 1.

Точка $\mathrm{U}_{3}: \lambda_{\gamma_{5}}$ и $\lambda_{\alpha_{1}}$ имеют индекс пересечения 0, т.е. гомологичны (с точностью до знака).

Другая идея, которую мы будем использовать в наших рассуждениях, состоит в следуюшем. На каждом атоме мы ввели допустимую систему координат. Поэтому мы можем рассмотреть эти системы координат на торе $T \in(1)$. Напомним [23], что ориентация базисных циклов согласована таким образом, что если мы рассмотрим матрицу перехода между теми из них, что соответствуют некоторому ребру изоэнергетической молекулы, то ее определитель будет равен -1 (другими словами, ориентация таких базисов противоположна). Это правило может быть переформулировано следующим образом. Если две бифуркации расположены обе слева (или обе справа) по отношению к области (1) (см. бифуркационные диаграммы на рис. 11), то ориентации соответствующих допустимых систем координат совпадают. В противном случае они противоположны.

Имеется еше одно полезное соображение, которое следует использовать в наших рассуждениях. Как уже было отмечено, иногда мы можем выбирать допустимую 
систему координат разньми способами для одного и того же атома. Например, в семействе (4) в качестве допустимой системы координат для $A \gamma_{4}^{\text {мы можем взять }}$ либо $\left(\lambda_{\gamma_{4}}, \lambda_{\beta_{2}}\right)$, либо $\left(\lambda_{\gamma_{4}}, \lambda_{\gamma_{3}}\right)$. Они имеют одну и ту же ориентацию. Заметим теперь, что для $l=0$ (см. рис. 11a)) имеется дополнительная симметрия, которая переставляет атомы $A \gamma_{4}{ }^{\text {и }} A \gamma_{1}$. Следовательно, то же самое утверждение остается справедливым и для атома $A \gamma_{\gamma_{1}}$ : базисы $\left(\lambda_{\gamma_{1}}, \lambda_{\beta_{2}}\right)$ и $\left(\lambda_{\gamma_{1}}, \lambda_{\gamma_{3}}\right)$ в семействе $(1)$ имеют одинаковую ориентацию.

Соберем теперь вместе всю информацию об ориентациях допустимых систем координат в семействе (1).

Базисы

$$
\left(\lambda_{\beta_{1}},-\lambda_{\gamma_{5}}\right),\left(\lambda_{\gamma_{5}}, \lambda_{\beta_{1}}\right),\left(\lambda_{\beta_{2}}, \lambda_{\gamma_{3}}\right),\left(\lambda_{\beta_{1}}, \lambda_{\gamma_{3}}+\lambda_{\beta_{1}}\right),\left(\lambda_{\gamma_{3}},-\lambda_{\beta_{2}}\right),\left(\lambda_{\gamma_{3}}, \lambda_{\beta_{1}}\right)
$$

имеют одинаковую ориентацию, тогда как $\left(\lambda_{\gamma_{1}}, \lambda_{\gamma_{3}}\right)$ и $\left(\lambda_{\gamma_{1}}, \lambda_{\beta_{2}}\right)$ ориентированы противоположным образом.

Теперь нетрудно убедиться в том, что перечисленные выше условия позволяют однозначно определить взаимное расположение всех циклов, относящихся к семейству (1). Результат показан на рис. 14a). Циклы $\lambda$ показаны здесь как элементы целочисленной решетки $Г$, отвечающей тору Лиувилля. Сам тор рассматривается как факторпространство $\mathbb{R}^{2} / \Gamma$.

Семейство (2).

Бифуркации, относящиеся к этому семейству, - это $A\left[\delta_{1}, B \beta_{1}, A^{*} \gamma_{2}, B \beta_{3}\right.$, $B_{\gamma_{6}}$

Особые точки, относящиеся к этому семейству, - это е $1, \mathrm{U}_{1}, \mathrm{~h}_{2}, \mathrm{U}_{2}, \mathrm{e}_{2}, \mathrm{M}_{2}$.

Информация об индексах пересечения, которая может быть получена из соответствующих круговых молекул, такова.

Точка $\mathrm{e}_{1}: \lambda_{\delta_{1}}$ и $\lambda_{\beta_{1}}$ имеют индекс пересечения 1.

Точка $\mathrm{U}_{1}: \lambda_{\beta_{1}}$ и $\lambda_{\gamma_{2}}$ имеют индекс пересечения 2.

Точка $\mathrm{h}_{2}: \lambda_{\gamma_{2}}$ и $\lambda_{\beta_{3}}$ имеют индекс пересечения 0 , т.е. гомологичны.

Точка $\mathrm{U}_{2}: \lambda_{\beta_{3}}$ и $\lambda_{\gamma_{6}}$ имеют индекс пересечения 1 , так же как $\lambda_{\beta_{1}}$ и $\lambda_{\gamma_{6}}$.

Точка е $2: \lambda_{\delta_{1}}$ и $\lambda_{\beta_{3}}$ имеют индекс пересечения 1.

Точка $\mathrm{M}_{2}: \lambda_{\delta_{1}}$ и $\lambda_{\gamma_{6}}$ имеют индекс пересечения 0 , т.е. гомологичны (с точностью до знака).

В этом семействе ориентации базисов

$$
\left(\lambda_{\beta_{1}},-\lambda_{\gamma_{6}}\right),\left(\lambda_{\gamma_{6}},-\lambda_{\beta_{3}}\right),\left(\lambda_{\gamma_{2}}, \frac{\lambda_{\beta_{1}}+\lambda_{\gamma_{2}}}{2}\right)
$$

совпадают и противоположны ориентации базисов

$$
\left(\lambda_{\delta_{1}}, \lambda_{\beta_{3}}\right),\left(\lambda_{\delta_{1}}, \lambda_{\beta_{1}}\right),\left(\lambda_{\gamma_{6}}, \lambda_{\beta_{1}}\right)
$$

Результат представлен на рис. 14б). 


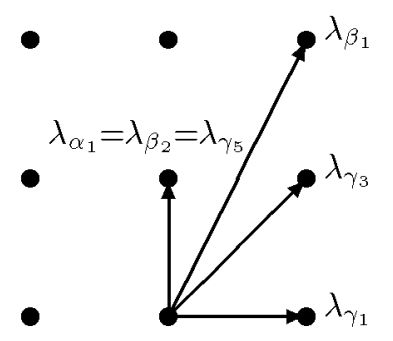

a) (1)

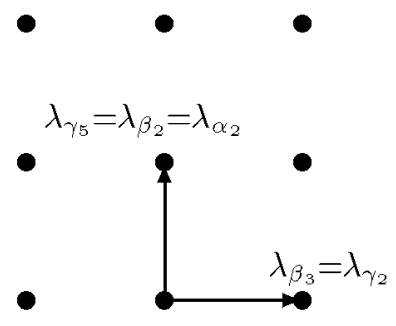

B)

(3)

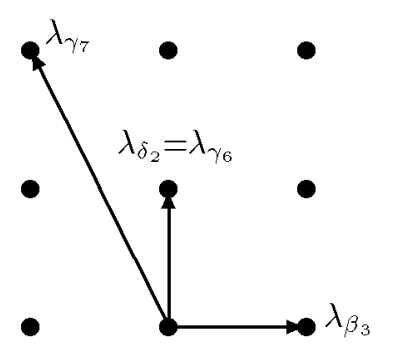

д)

(5)

Рис. 14

\section{Семейство (3).}

Бифуркации, относящиеся к этому семейству, -это $A_{\gamma_{2}}^{*}, C_{2} \beta_{2}, B \gamma_{5}, B \beta_{3}$, ${ }^{A} \alpha_{2}$.

Особые точки, относящиеся к этому семейству, - это $\mathrm{U}_{1}, \mathrm{~h}_{1}, \mathrm{U}_{2}, \mathrm{~h}_{2}, \mathrm{U}_{3}, \mathrm{e}_{2}$.

Информация, которая извлекается из соответствующих круговых молекул, такова.

Точка $\mathrm{U}_{1}: \lambda_{\gamma_{2}}$ и $\lambda_{\beta_{2}}$ имеют индекс пересечения 1.

Точка $\mathrm{h}_{1}: \lambda_{\beta_{2}}$ и $\lambda_{\gamma_{5}}$ имеют индекс пересечения 0 , т.е. гомологичны.

Точка $\mathrm{U}_{2}: \lambda_{\gamma_{5}}$ и $\lambda_{\beta_{3}}$ имеют индекс пересечения 1. 
Точка $\mathrm{h}_{2}: \lambda_{\gamma_{2}}$ и $\lambda_{\beta_{3}}$ имеют индекс пересечения 0 , т.е. гомологичны.

Точка $\mathrm{U}_{3}: \lambda_{\alpha_{2}}$ и $\lambda_{\gamma_{5}}$ имеют индекс пересечения 0, т.е. гомологичны (с точностью до знака).

Точка $e_{2}: \lambda_{\alpha_{2}}$ и $\lambda_{\beta_{3}}$ имеют индекс пересечения 1.

Информация об ориентациях допустимых систем координат на торах из семейства (3) такова.

Базисы

$$
\left(\lambda_{\beta_{3}},-\lambda_{\gamma_{5}}\right),\left(\lambda_{\gamma_{2}},-\lambda_{\beta_{2}}\right)
$$

имеют одинаковую ориентацию, а ориентация базисов

$$
\left(\lambda_{\gamma_{5}},-\lambda_{\beta_{3}}\right),\left(\lambda_{\beta_{2}},-\lambda_{\gamma_{2}}\right),\left(\lambda_{\alpha_{2}}, \lambda_{\beta_{3}}\right)
$$

ей противоположна.

Результат снова однозначен и показан на рис. 14в).

Семейство (4).

Бифуркации, относящиеся к этому семейству, $-C_{2} \beta_{2}, B \gamma_{3}, A\left[\gamma_{4}\right.$.

Особые точки, относящиеся к этому семейству, - это $\mathrm{U}_{1}, \mathrm{~h}_{1}, \mathrm{c}_{1}$.

Точка $\mathrm{U}_{1}: \lambda_{\beta_{2}}$ и $\lambda_{\gamma_{3}}$ имеют индекс пересечения 1.

Точка $\mathrm{h}_{1}: \lambda_{\beta_{2}}$ и $\lambda_{\gamma_{4}}$ имеют индекс пересечения 1 .

Точка с 1 : $\lambda_{\gamma_{3}}$ и $\lambda_{\gamma_{4}}$ имеют индекс пересечения 1.

Ситуация с ориентациями довольна проста.

Базисы

$$
\left(\lambda_{\gamma_{4}}, \lambda_{\gamma_{3}}\right),\left(\lambda_{\gamma_{4}}, \lambda_{\beta_{2}}\right)
$$

имеют одинаковую ориентацию, причем эта ориентация противоположна ориентации пары $\left(\lambda_{\beta_{2}}, \lambda_{\gamma_{3}}\right)$.

Конечный результат показан на рис. $14 \Gamma)$.

Семейство (5).

Бифуркации, относящиеся к этому семейству, -это $B\left[\beta_{3}, B_{\gamma_{6}}, A_{\gamma_{7}}, A\left[\delta_{2}\right.\right.$.

Особые точки, относящиеся к этому семейству, - это $\mathrm{U}_{2}, \mathrm{~h}_{2}, \mathrm{c}_{2}, \mathrm{M}_{1}, \mathrm{M}_{2}, \mathrm{e}_{2}$.

Информация, извлекаемая из особых точек, такова.

Точка $\mathrm{U}_{2}: \lambda_{\beta_{3}}$ и $\lambda_{\gamma_{6}}$ имеют индекс пересечения 1.

Точка $\mathrm{h}_{2}: \lambda_{\beta_{3}}$ и $\lambda_{\gamma_{7}}$ имеют индекс пересечения 2.

Точка с $2: \lambda_{\gamma_{6}}$ и $\lambda_{\gamma_{7}}$ имеют индекс пересечения 1.

Точка $\mathrm{M}_{1}: \lambda_{\delta_{2}}$ и $\lambda_{\gamma_{7}}$ имеют индекс пересечения 1.

Точка $\mathrm{M}_{2}: \lambda_{\gamma_{6}}$ и $\lambda_{\delta_{2}}$ имеют индекс пересечения 0, т.е. гомологичны (с точностью до знака).

Точка е $2: \lambda_{\delta_{2}}$ и $\lambda_{\beta_{3}}$ имеют индекс пересечения 1.

Ориентация базиса

$$
\left(\lambda_{\beta_{3}}, \lambda_{\gamma_{6}}\right)
$$

противоположна ориентации базисов

$$
\left(\lambda_{\delta_{2}}, \lambda_{\beta_{3}}\right),\left(\lambda_{\gamma_{7}}, \lambda_{\beta_{3}}\right),\left(\lambda_{\gamma_{7}}, \lambda_{\gamma_{6}}\right)
$$

Результат показан на рис. 14 д). 
4.3. Матрицы склейки. Теперь нам известно все о базисных циклах допустимых систем координат, и мы можем выписать все матрицы склейки, соответствуюшие каждой из десяти изоэнергетических молекул.

Рассмотрим, например, молекулу, отвечаюшую уровню энергии $D$ на рис. 10б):

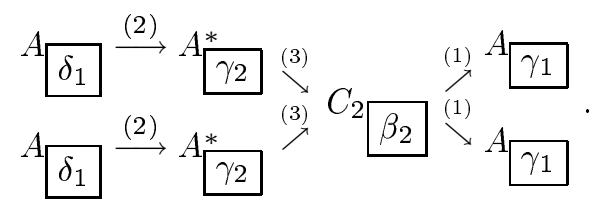

Для каждого из шести ребер этой молекулы мы восстанавливаем матрицу склейки. Отметим, что каждое ребро соответствует некоторому семейству торов, поэтому мы должны лишь извлечь требуемую информацию из рис. 14. А именно,

$$
\begin{aligned}
\left(\begin{array}{c}
\lambda_{\gamma_{1}} \\
\lambda_{\gamma_{3}}
\end{array}\right) & =C_{\gamma_{1}, \beta_{2}}\left(\begin{array}{c}
\lambda_{\beta_{2}} \\
\lambda_{\gamma_{3}}
\end{array}\right)=\left(\begin{array}{cc}
-1 & 1 \\
0 & 1
\end{array}\right)\left(\begin{array}{l}
\lambda_{\beta_{2}} \\
\lambda_{\gamma_{3}}
\end{array}\right), \\
\left(\begin{array}{c}
\lambda_{\beta_{2}} \\
-\lambda_{\gamma_{2}}
\end{array}\right) & =C_{\beta_{2}, \gamma_{2}}\left(\begin{array}{c}
\lambda_{\gamma_{2}} \\
-\lambda_{\beta_{2}}
\end{array}\right)=\left(\begin{array}{cc}
0 & -1 \\
-1 & 0
\end{array}\right)\left(\begin{array}{c}
\lambda_{\gamma_{2}} \\
-\lambda_{\beta_{2}}
\end{array}\right), \\
\left(\begin{array}{c}
\lambda_{\gamma_{2}} \\
\frac{\lambda_{\beta_{1}}+\lambda_{\gamma_{2}}}{2}
\end{array}\right) & =C_{\gamma_{2}, \delta_{1}}\left(\begin{array}{c}
\lambda_{\delta_{1}} \\
\lambda_{\beta_{1}}
\end{array}\right)=\left(\begin{array}{ll}
-2 & 1 \\
-1 & 1
\end{array}\right)\left(\begin{array}{c}
\lambda_{\delta_{1}} \\
\lambda_{\beta_{1}}
\end{array}\right) .
\end{aligned}
$$

В табл. 5 мы собрали информацию о матрицах склейки для каждого из десяти возможных типов слоений Лиувилля на изоэнергетических поверхностях.

4.4. Числовые метки. В терминах работы [11] совокупность матриц склеек называется избыточным оснащением молекулы. Избыточность означает, что мы имеем больше информации, чем необходимо для определения топологии слоения (она отражает тот факт, что допустимые системы координат и, следовательно, матрицы склейки не определены однозначно). В [11], [23] была предложена процедура, которая сокрашает имеюшуюся информацию и делает ее однозначной. Вместо матриц склейки следует рассмотреть некоторые числовые метки $(r, \varepsilon, n)$, которые удовлетворяют следующим естественным свойствам:

1) они вычисляются однозначно по матрицам склейки,

2) они не меняются при заменах допустимых систем координат,

3) матрицы склейки могут быть однозначно восстановлены по этим меткам с точностью до некоторой замены допустимых систем координат.

Таким образом, заменяя матрицы склейки на эти числовые метки, мы не теряем никакой важной информации, а лишш делаем ее однозначно определенной.

Эта процедура завершает доказательство. Определение и формальные правила для вычисления $(r, \varepsilon, n)$ содержатся в [13], [23]. Здесь эти вычисления опушены. Их результатом является в точности утверждение теоремы (см. табл. 3 ). 
ТАБЛИцА 5. Матрицы склейки

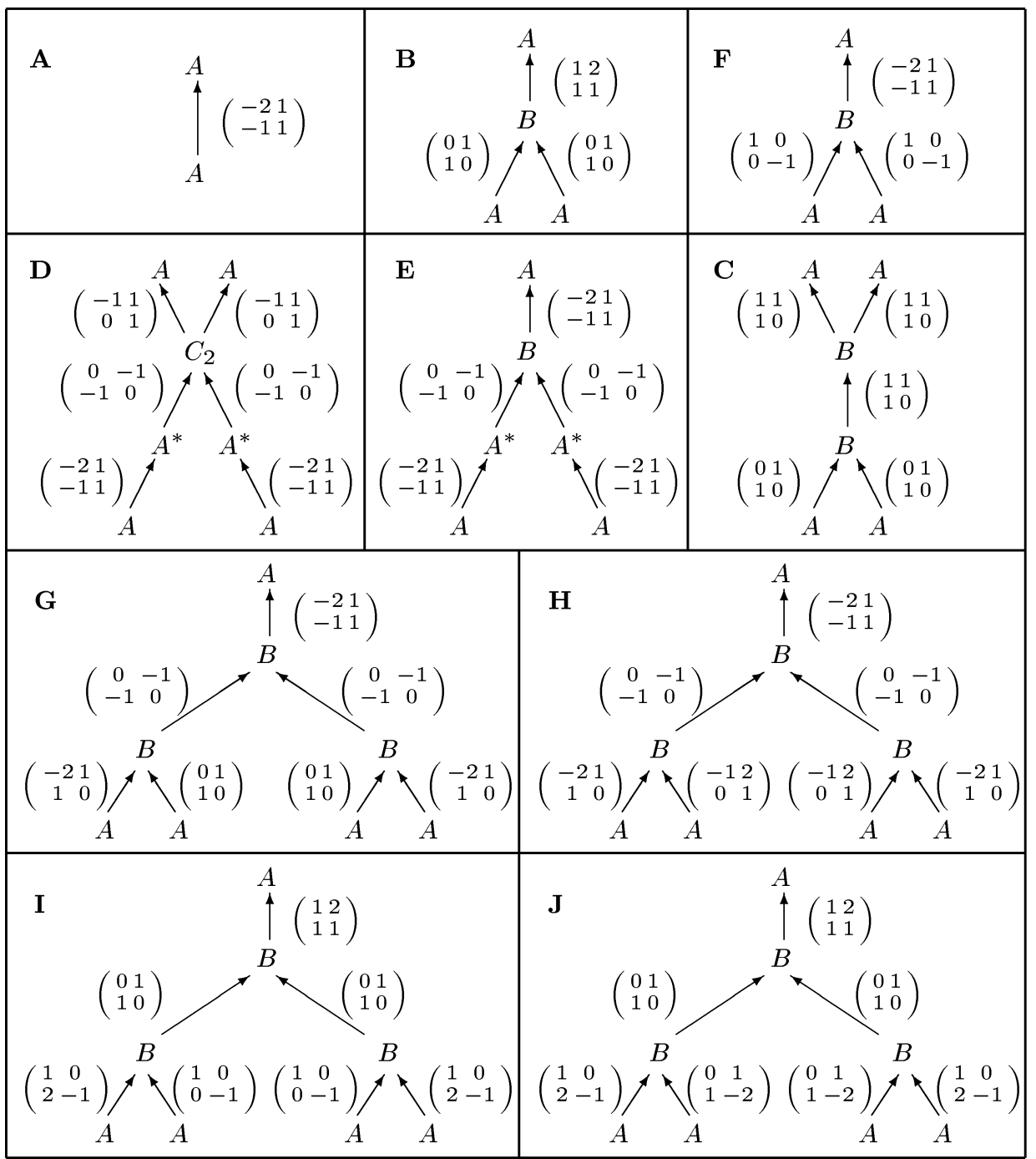

\section{Список литературы}

1. Kowalevski S. Sur le probléme de la rotation d'un corps solide autour d'un point fixe // Acta Math. 1889. V. 12. Р. 177-232; Русский перевод: Ковалевская С. В. Задача о вращении твердого тела около неподвижной точки // Ковалевская С. В. Научные работы. М.: Изд-во АН СССР, 1948. С. 153-220.

2. Cooke $R$. The Mathematics of Sonya Kovalevskaya. Berlin: Springer-Verlag, 1984.

3. Kowalevski $S$. Sur une propriété du systéme d'équations différentielles qui définit la rotation d'un corps solide d'un point fixe // Acta Math. 1889. V. 14. P. 81-93.

4. Klein F., Sommerfeld A. Über die Theorie des Kreisels. Leipzig: Teubner, 1910.

5. Kötter F. Sur le cas traité par Mme Kowalevski de rotation d'un corps solide autour d'un point fixe // Acta Math. 1893. V. 17. P. 209-264.

6. Bobenko A.I., Reyman A.G., Semenov-Tian-Shansky M. A. The Kowalevski top 99 years later: a Lax pair, generalizations and explicit solutions // Comm. Math. Phys. 1989. V. 122. P. 321-354. 
7. Жуковский H. E. Геометрическая интерпретация случая движения тяжелого твердого тела вокруг фиксированной точкой, рассмотренного Ковалевской // Матем. сб. 1896. T. 19 . C. $45-93$.

8. Anпельрот Г. Г. Не вполне симметричные тяжелые гироскопы // Движение твердого тела вокруг неподвижной точки. М.-Л.: Изд-во АН СССР, 1940. С. 61-157.

9. Харламов М.П. Бифуркации совместных поверхностей уровня первых интегралов в задаче Ковалевской // Прикл. матем. и мех. 1983. Т. 47. №6. С. 737-743.

10. Fomenko A. T. Topological classification of all integrable Hamiltonian differential equations of general type with two degrees of freedom // The geometry of Hamiltonian systems / ed. T. Ratiu. New York: Springer-Verlag, 1991. P. 131-339.

11. Болсинов A.B., Матвеев C.В., Фоменко A.T. Топологическая классификация интегрируемых гамильтоновых систем с двумя степенями свободы. Список систем малой сложности // УМН. 1990. Т. 45. № 2. С. 49-77.

12. Oshemkov A. A. Fomenko invariants for the main integrable cases of the rigid body motion equations // Topological classification of integrable systems. Adv. Soviet Math. / ed. A.T. Fomenko. V. 6. Providence, RI: Amer. Math. Soc., 1991. P. 67-146.

13. Болсинов A. В., Фоменко A. T. Введение в топологию интегрируемых гамилтоновых систем. М.: Наука, 1997.

14. Топалов П. Вычисление тонкого инварианта Фоменко-Цишанга для основных интегрируемых случаев движения твердого тела // Матем. сб. 1996. Т. 187. №3. С. 143-160.

15. Dullin H. R. Die Energieflächen des Kowalewskaja-Kreisels. Dissertation Univ. Bremen. Aachen: Mainz Verlag, 1994.

16. Dullin H.R., Juhnke M., Richter P. H. Action integrals and energy surfaces of the Kovalevskaya top // Internat. J. Bifur. Chaos Appl. Sci. Engrg. 1994. V. 4. №6. P. 1535-1562.

17. Dullin H.R., Richter P. H., Veselov A.P. Action variables of the Kovalevskaya top // Regular and Chaotic Dynam. 1998. V. 3. № 3. P. 18-31.

18. Adler M., van Moerbeke $P$. The Kowalewski and Henon-Heiles motions as Manakov geodesic flows on SO(4). A two-dimensional family of Lax pairs // Comm. Math. Phys. 1988. V. 113. № 4. P. 659-700.

19. Adler M., van Moerbeke P. Kowalewski's asymptotic method, Kac-Moody algebras, and regularizations // Comm. Math. Phys. 1982. V. 83. P. 83-106.

20. Audin M., Silhol R. Variétés Abéliennes Réelles et Toupie de Kowalewski // Compositio Math. 1993. V. 87. P. 153-229.

21. Yahia H. M. New integrable cases in dynamics of rigid bodies // Mech. Res. Comm. 1986. V. 13. №3. P. 169-172.

22. Ольианецкий М.А., Переломов А.М., Рейман А.Г., Семенов-Тян-Шанский M. A. Интегрируемые системы. II // Итоги науки и техники. Совр. проблемы матем. Фундам. направления. Т. 16. М.: ВИНИТИ, 1987. С. 86-226.

23. Болсинов A.B., Фоменко A.T. Интегрируемые гамильтоновы системы. Геометрия. Топология. Классификация. Т. 1, 2. Ижевск: Издательский дом "Удмуртский университет", 1999.

24. Лерман Л. М., Уманский $Я$. Л. Классификация четырехмерных интегрируемых гамильтоновых систем и пуассоновских действий $\mathbb{R}^{2}$ в расширенных окрестностях простых особых точек. I, II, III // Матем. сб. 1992. Т. 183. № 12. С. 141-176; 1993. Т. 184. № 4 . С. $105-138 ;$ 1995. Т. 186. № 10. С. 89-102.

25. Матвеев B. С. Интегрируемые гамильтоновы системы с двумя степенями свободы. Топологическое строение насьпщенных окрестностей точек типа фокус-фокус и седло-седло // Матем. сб. 1996. Т. 187. № 4. С. 29-58.

26. Nguen Tien Zung. Decomposition of nondegenerate singularities of integrable Hamiltonian systems // Lett. Math. Phys. 1995. V. 33. № 3. P. 187-193.

27. Bolsinov A. V. Methods of calculation of the Fomenko-Zieschang invariant // Topological classification of integrable systems. Adv. Soviet Math. / ed. A.T. Fomenko. V. 6. Providence, RI: Amer. Math. Soc., 1991. P. 147-183. 
28. Харламов М. П. Топологический анализ интегрируемых задач динамики твердого тела. Л.: Изд-во ЛГУ, 1988.

29. Audin M. Spinning tops. A course on integrable systems. Cambridge: Cambridge Univ. Press, 1996.

30. Cushman R.H., Bates L.M. Global aspects of classical integrable systems. Basel: Birkhäuser, 1997.

31. Калашников В. В. (мл.). Типичные интегрируемые гамильтоновы системы на четырехмерном симплектическом многообразии // Изв. РАН. Сер. матем. 1998. Т. 62. № 2. C. $49-74$.

32. Richter P.H., Dullin H.R., Wittek A. Kovalevskaya Top. Film C1961 // Publ. Wiss. Film., Techn. Wiss./ Naturw. V. 13. Göttingen: Inst. Wiss. Film, 1997. P. 33-96.

Московский государственньй

Поступила в редакцию

университет им. М.В. Ломоносова;

28.10.1999

Институт теоретической физики

Бременского университета, Германия 